\title{
A formalism for the calculus of variations with spinors
}

\author{
Thomas Bäckdahl' ${ }^{1, a)}$ and Juan A. Valiente Kroon ${ }^{2, b)}$ \\ ${ }^{1}$ The School of Mathematics, University of Edinburgh, JCMB 6228, Peter Guthrie Tait Road, \\ Edinburgh EH9 3FD, United Kingdom and Mathematical Sciences - Chalmers University \\ of Technology and University of Gothenburg - SE-412 96 Gothenburg, Sweden \\ ${ }^{2}$ School of Mathematical Sciences, Queen Mary, University of London, Mile End Road, \\ London E1 4NS, United Kingdom
}

(Received 19 May 2015; accepted 23 December 2015; published online 12 January 2016)

\begin{abstract}
We develop a frame and dyad gauge-independent formalism for the calculus of variations of functionals involving spinorial objects. As a part of this formalism, we define a modified variation operator which absorbs frame and spin dyad gauge terms. This formalism is applicable to both the standard spacetime (i.e., $S L(2, \mathbb{C})$ ) 2-spinors as well as to space (i.e., $S U(2, \mathbb{C})) 2$-spinors. We compute expressions for the variations of the connection and the curvature spinors. ( 2016 AIP Publishing LLC. [http://dx.doi.org/10.1063/1.4939562]
\end{abstract}

\section{INTRODUCTION}

Variational ideas play an important role in various areas of mathematical general relativitye.g., in the ADM (Arnowitt, Deser, Misner) formalism, ${ }^{1}$ in the analysis of Penrose-like inequalities, ${ }^{8}$ or in the analysis of area-angular momentum inequalities ${ }^{6}$ to mention some. Similarly, spinorial methods constitute a powerful tool for the analysis and manipulation of the Einstein field equations and their solutions-most notably the proof of the positivity of the mass by Witten ${ }^{13}$ and the analysis of linearised gravity, see, e.g., Ref. 10.

To the best of our knowledge, all available treatments of calculus of variations and linerisations in spinorial settings make use of computations in terms of components with respect to a dyad. It is therefore of interest to have a setup for performing a dyad-independent calculus of variations and computation of linearisations with spinors. The purpose of the present article is to develop such a setup. We expect this formalism to be of great value in both the analysis of the notion of non-Kerrness introduced in Refs. 3 and 4 and positivity of the mass in Ref. 5, as well as in a covariant analysis of linearised gravity.

The transformation properties of tensors and spinors pose some conceptual subtleties which have to be taken into account when computing variations of the basic tensorial and spinorial structures. It is possible to have variations of these structures which are pure gauge. This difficulty is usually dealt with by a careful fixing of the gauge in some geometrically convenient manner. One thus makes calculus of variations in a specific gauge and has to be careful in distinguishing between properties which are specific to the particular gauge and those which are generic. This situation becomes even more complicated as, in principle, both the tensorial and spinorial structures are allowed to vary simultaneously.

In this article, it is shown that it is possible to define a modified variation operator which absorbs gauge terms in the variation of spinorial fields and thus allows to perform covariant variations. The idea behind this modified variation operator is similar to that behind the derivative operators in the GHP (Geroch, Held, Penrose) formalism which absorb terms associated to the freedom in a NP (Newman, Penrose) tetrad-see Ref. 7. As a result of our analysis, we are able to obtain expressions involving abstract tensors and spinors-thus, they are valid in any system of coordinates, and therefore invariant under diffeomorphisms which are constant with respect to

\footnotetext{
a)E-mail address: thobac@chalmers.se

b) E-mail address: j.a.valiente-kroon@qmul.ac.uk
} 
variations. However, linearisations of diffeomorphisms do affect our variational quantities. This is discussed in Section IV C, where we also find that the diffeomorphism freedom can be controlled by a gauge source function.

Finally, we point out that although our primary concern in this article is the construction of a formalism for the calculus of variations of expressions involving spinors in a 4-dimensional Lorentzian manifold, the methods can be adapted to a space-spinor formalism on 3-dimensional Riemannian manifolds. This is briefly discussed in Section VI.

The calculations in this article have been carried out in the Mathematica based symbolic differential geometry suite $x A c t,{ }^{9}$ in particular, SymManipulator ${ }^{2}$ developed by TB.

\section{A. Notation and conventions}

Throughout, we use abstract index notation to denote tensors and spinors. In particular, the indices $a, b, c, \ldots$ and $i, j, k, \ldots$ are abstract spacetime and spatial tensor indices, respectively, while $A, B, C, \ldots$ denote abstract spinorial indices. The boldface indices $\mathbf{a}, \mathbf{b}, \mathbf{c}, \ldots$ and $\mathbf{A}, \mathbf{B}, \mathbf{C}, \ldots$ will be used as tensor frame indices and spinor frame indices, respectively. We follow the tensorial and spinorial conventions of Penrose and Rindler. ${ }^{10}$

Our signature convention for 4-dimensional Lorentzian metrics is $(+,-,-,-)$, and 3-dimensional Riemannian metrics have signature $(-,-,-)$.

The standard positions for the basic variations are $\delta g_{a b}, \delta \sigma_{a}{ }^{A A^{\prime}}, \delta \sigma_{k}{ }^{A B}, \delta \omega^{\mathbf{a}}{ }_{b}, \delta \epsilon^{\mathbf{A}}{ }_{B}, \delta \epsilon_{A B}$, $\delta \gamma_{a}{ }^{b}{ }_{c}$. If any other index positions appear, this means that the indices are moved up or down with $g_{a b}$ or $\epsilon_{A B}$ after the variation. The definitions of the above objects will be given in the main text.

\section{BASIC SETUP}

In this section, we discuss our basic geometric setup, which will be used in Section III to perform calculus of variations.

\section{A. Families of metrics}

In what follows, let $\left(\mathcal{M}, \stackrel{\circ}{g}_{a b}\right)$ denote a 4-dimensional Lorentzian manifold (spacetime). The metric $\stackrel{\circ}{g}_{a b}$ will be known as the background metric. In what follows, in addition to $\stackrel{\circ}{a}_{a b}$, we consider arbitrary families of Lorentzian metrics $\left\{g_{a b}[\lambda]\right\}$ over $\mathcal{M}$ with $\lambda \in \mathbb{R}$ a parameter such that $g_{a b}[0]=\stackrel{\circ}{g}_{a b}$. Intuitively, a particular choice of family of metrics can be thought of as a curve in the moduli space of Lorentzian metrics over $\mathcal{M}$. The fact that we allow for arbitrary families of metrics enables us to probe all possible directions of this space in a neighbourhood of $\stackrel{\circ}{g}_{a b}$ and thus, we can compute Fréchet derivatives of functionals depending on the metric-see Section III A.

In order to make possible the discussion of spinors, it will be assumed that the spacetimes $\left(\mathcal{M}, g_{a b}[\lambda]\right)$ for fixed $\lambda$ are orientable and time orientable and admit a spinorial structure.

Notational warning. In what follows, for the ease of the presentation, we often suppress the dependence on $\lambda$ from the various objects. Thus, unless otherwise stated, all objects not tagged with a ring $\left({ }^{\circ}\right)$ are assumed to depend on a parameter $\lambda$.

\section{B. Frames}

In what follows, we assume that associated to each family of metrics $\left\{g_{a b}\right\}$, one has a family $\left\{e_{\mathbf{a}}{ }^{a}\right\}$ of $g_{a b}$-orthonormal frames. Let $\left\{\omega^{\mathbf{a}}{ }_{a}\right\}$ denote the family of associated cobases so that for fixed $\lambda$ one has $e_{\mathbf{a}}{ }^{a} \omega^{\mathbf{b}}{ }_{a}=\delta_{\mathbf{a}}{ }^{\mathbf{b}}$. Following the conventions of Sec. II A, we write $\check{e}_{\mathbf{a}}{ }^{a} \equiv e_{\mathbf{a}}{ }^{a}[0]$ and $\stackrel{\circ}{\omega}_{a}^{\mathbf{a}} \equiv \omega^{\mathbf{a}}{ }_{a}[0]$. By assumption, one has that

$$
g_{a b} e_{\mathbf{a}}{ }^{a} e_{\mathbf{b}}{ }^{b}=\eta_{\mathbf{a b}}, \quad g_{a b}=\eta_{\mathbf{a b}} \omega^{\mathbf{a}}{ }_{a} \omega_{b}^{\mathbf{b}},
$$

where, as usual, $\eta_{\mathbf{a b}}=\operatorname{diag}(1,-1,-1,-1)$. 
Remark 1. Observe that in view of relations (1), any family of frames and coframes $\left\{e_{\mathbf{a}}^{\prime a}\right\}$ and $\left\{\omega^{\prime \mathbf{a}}{ }_{a}\right\}$ related to $\left\{e_{\mathbf{a}}{ }^{a}\right\}$ and $\left\{\omega^{\mathbf{a}}{ }_{a}\right\}$ through a family of Lorentz transformations $\left\{\Lambda_{\mathbf{b}}^{\mathbf{a}}\right\}$ give rise to the same family of metrics $\left\{g_{a b}\right\}$-see Appendix A 1 .

\section{Spinors}

By assumption, the spacetimes $\left(\mathcal{M}, g_{a b}\right)$ are endowed with a spinorial structure. Accordingly, we consider families of antisymmetric spinors $\left\{\epsilon_{A B}\right\}$ such that for fixed $\lambda$, the spinor $\epsilon_{A B}$ gives rise to the spinor structure of $\left(\mathcal{M}, g_{a b}\right)$. Moreover, we set $\stackrel{\circ}{\epsilon B}_{A B} \equiv \epsilon_{A B}[0]$.

Associated to the family $\left\{\epsilon_{A B}\right\}$, one considers a family $\left\{\epsilon_{\mathbf{A}}{ }^{A}\right\}$ of normalised spin dyads, that is, one has that

$$
\epsilon_{A B} \epsilon_{\mathbf{A}}^{A} \epsilon_{\mathbf{B}}^{B}=\epsilon_{\mathrm{AB}}, \quad \epsilon_{\mathrm{AB}} \equiv\left(\begin{array}{cc}
0 & 1 \\
-1 & 0
\end{array}\right) .
$$

Let $\left\{\epsilon^{\mathbf{A}}{ }_{A}\right\}$ denote the family of dual covariant bases for which the relation $\epsilon^{A B} \epsilon^{\mathbf{A}}{ }_{A} \epsilon^{\mathbf{B}}{ }_{B}=\epsilon^{\mathbf{A B}}$ with $\left(\epsilon^{\mathbf{A B}}\right) \equiv-\left(\epsilon_{\mathbf{A B}}\right)^{-1}$ holds. It follows that one has

$$
\delta_{A}{ }^{B}=\epsilon_{A}{ }^{\mathbf{A}} \epsilon_{\mathbf{A}}{ }^{B}, \quad \epsilon_{A B}=\epsilon_{\mathbf{A B}} \epsilon_{A}{ }^{\mathbf{A}} \epsilon_{B}{ }^{\mathbf{B}}, \quad \epsilon^{A B}=\epsilon^{\mathbf{A B}} \epsilon_{\mathbf{A}}{ }^{A} \epsilon_{\mathbf{B}}{ }^{B} .
$$

Remark 2. As in the case of tensor frames, any family of dyads $\left\{\epsilon_{\mathbf{A}}^{\prime}{ }^{A}\right\}$ related to $\left\{\epsilon_{\mathbf{A}}{ }^{A}\right\}$ through a family of Lorentz transformations $\left\{\Lambda^{\mathbf{A}}{ }_{\mathbf{B}}\right\}$ gives rise to the same spinorial structures associated to the family of antisymmetric spinors $\left\{\epsilon_{A B}\right\}$-see Appendix $A 1$.

\section{Infeld-van der Waerden and soldering forms}

The well-known correspondence between tensors and spinors is realised by the Infeld-van der Waerden symbols $\sigma_{\mathbf{a}}{ }^{\mathbf{A A ^ { \prime }}}$ and $\sigma^{\mathbf{a}}{ }_{\mathbf{A A}}$. Given an arbitrary $v^{a} \in T \mathcal{M}$ and $\beta_{a} \in T^{*} \mathcal{M}$, one has that

$$
v^{\mathbf{a}} \mapsto v^{\mathbf{A A}^{\prime}}=v^{\mathbf{a}} \sigma_{\mathbf{a}}^{\mathbf{A A}^{\prime}}, \quad \beta_{\mathbf{a}} \mapsto \beta_{\mathbf{A A}^{\prime}}=\beta_{\mathbf{a}} \sigma^{\mathbf{a}} \mathbf{A A}^{\prime},
$$

where for fixed $\lambda$,

$$
v^{\mathbf{a}} \equiv v^{a} \omega_{a}^{\mathbf{a}}, \quad \beta_{\mathbf{a}} \equiv \beta_{a} e_{\mathbf{a}}^{a}
$$

denote the components of $v^{a}$ and $\beta_{a}$ with respect to the orthonormal basis $e_{\mathbf{a}}{ }^{a}[\lambda]$ of $\left(\mathcal{M}, g_{a b}[\lambda]\right)$. In more explicit terms, the correspondence can be written as

$$
\left(v^{0}, v^{1}, v^{2}, v^{3}\right) \mapsto \frac{1}{\sqrt{2}}\left(\begin{array}{cc}
v^{0}+v^{3} & v^{1}+\mathrm{i} v^{2} \\
v^{1}-\mathrm{i} v^{2} & v^{0}-v^{3}
\end{array}\right),\left(\beta_{0}, \beta_{1}, \beta_{2}, \beta_{3}\right) \mapsto \frac{1}{\sqrt{2}}\left(\begin{array}{cc}
\beta_{0}+\beta_{3} & \beta_{1}-\mathrm{i} \beta_{2} \\
\beta_{1}+\mathrm{i} \beta_{2} & \beta_{0}-\beta_{3}
\end{array}\right) .
$$

From the Infeld-van der Waerden symbols, we define the soldering form $\sigma_{a}{ }^{A A^{\prime}}$ and the dual of the soldering form $\sigma^{a}{ }_{A A^{\prime}}$ by

$$
\begin{aligned}
\sigma_{a}{ }^{A A^{\prime}} & \equiv \epsilon_{\mathbf{A}}{ }^{A} \bar{\epsilon}_{\mathbf{A}^{\prime}}{ }^{A^{\prime}} \omega^{\mathbf{a}}{ }_{a} \sigma_{\mathbf{a}}{ }^{\mathbf{A A}^{\prime}}, \\
\sigma^{a}{ }_{A A^{\prime}} & \equiv \epsilon^{\mathbf{A}}{ }_{A} \bar{\epsilon}^{\mathbf{A}^{\prime}{ }_{A^{\prime}} e_{\mathbf{a}}{ }^{a} \sigma^{\mathbf{a}}{ }_{\mathbf{A} \mathbf{A}^{\prime}} .}
\end{aligned}
$$

By direct calculation, we can then verify the relations

$$
\begin{aligned}
& g_{a b}=\epsilon_{A B} \bar{\epsilon}_{A^{\prime} B^{\prime}} \sigma_{a}{ }^{A A^{\prime}} \sigma_{b}{ }^{B B^{\prime}}, \\
& \delta_{a}{ }^{b}=\sigma_{a}{ }^{B B^{\prime}} \sigma^{b}{ }_{B B^{\prime}} .
\end{aligned}
$$

It is important to note that $\sigma_{a}{ }^{A A^{\prime}}$ and $\sigma^{a}{ }_{A A^{\prime}}$ are tensor frame and spin dyad dependent, while relations (4a) and (4b) are universal.

Following our approach, in the sequel, we consider families $\left\{\sigma_{a}{ }^{A A^{\prime}}\right\}$ and $\left\{\sigma^{a}{ }_{A A^{\prime}}\right\}$ of soldering forms such that $\stackrel{\circ}{\sigma}_{a} A A^{\prime} \equiv \sigma_{a}{ }^{A A^{\prime}}[0]$ and $\stackrel{\circ}{\sigma}^{a}{ }_{A A^{\prime}} \equiv \sigma^{a}{ }_{A A^{\prime}}[0]$ are the soldering forms associated to $\left(\stackrel{\circ}{\omega}_{a}^{\mathbf{b}}, \stackrel{\circ}{\epsilon}_{A}^{\mathbf{B}}\right)$. 
Remark 3. In this article, we adopt the point of view that the metric structure provided by $g_{a b}$ and the spinorial structure given by $\epsilon_{A B}$ are independent from each other. After a choice of frame and spinor basis, these structures are linked to each other-in an, admittedly, arbitrary manner-through the relations in (3a) and (4a).

\section{CALCULUS OF VARIATIONS}

\section{A. Basic formalism}

The main objective of our calculus of variations is to describe how real valued functionals depend on their arguments-in particular, in the case, the arguments are covariant spinors. To motivate our analysis, we first consider a real valued functional $\mathcal{F}\left[\omega^{\mathbf{a}}{ }_{a}, \xi^{\mathbf{a}}\right]$, where $\xi^{a}$ is a vector field and $\xi^{\mathbf{a}}=\omega^{\mathbf{a}}{ }_{a} \xi^{a}$. Given a particular family of fields $\left\{\omega^{\mathbf{a}}{ }_{a}[\lambda], \xi^{\mathbf{a}}[\lambda]\right\}$ depending on a parameter $\lambda$, we define the variations $\left\{\delta \omega^{\mathbf{a}}{ }_{a}, \delta \xi^{\mathbf{a}}\right\}$ through the expressions

$$
\left.\delta \omega^{\mathbf{a}}{ }_{a} \equiv \frac{\mathrm{d} \omega^{\mathbf{a}}{ }_{a}}{\mathrm{~d} \lambda}\right|_{\lambda=0},\left.\quad \delta \xi^{\mathbf{a}} \equiv \frac{\mathrm{d} \xi^{\mathbf{a}}}{\mathrm{d} \lambda}\right|_{\lambda=0} .
$$

In terms of the above fields over $\mathcal{M}$, we define the Gâteux derivative of $\mathcal{F}\left[\omega^{\mathbf{a}}{ }_{a}, \xi^{\mathbf{a}}\right]$ at $\left\{\dot{\circ}^{\mathbf{a}}{ }_{a}, \dot{\ell}^{\mathbf{a}}\right\}$ in the direction of the family $\left\{\omega^{\mathbf{a}}{ }_{a}[\lambda], \xi^{\mathrm{a}}[\lambda]\right\}$ as

$$
\begin{aligned}
\delta_{\left\{\omega_{a}{ }_{a}, \xi \mathbf{a}\right\}} \mathcal{F}\left[\stackrel{\circ}{\mathbf{a}}_{a}, \check{\xi}^{\mathbf{a}}\right] & \left.\equiv \frac{\mathrm{d}}{\mathrm{d} \lambda} \mathcal{F}\left[\omega^{\mathbf{a}}{ }_{a}[\lambda], \xi^{\mathbf{a}}[\lambda]\right]\right|_{\lambda=0} \\
& =\left.\frac{\mathrm{d}}{\mathrm{d} \lambda} \mathcal{F}\left[\stackrel{\circ}{\omega}^{\mathbf{a}}{ }_{a}+\lambda \delta \omega^{\mathbf{a}}{ }_{a}, \stackrel{\xi}{\xi}^{\mathbf{a}}+\lambda \delta \xi^{\mathbf{a}}\right]\right|_{\lambda=0} .
\end{aligned}
$$

Now, if $\delta_{\left\{\omega^{\mathbf{a}}{ }_{a}, \xi^{\mathbf{a}}\right\}} \mathcal{F}$ exists for any choice of family $\left\{\omega^{\mathbf{a}}{ }_{a}, \xi^{\mathbf{a}}\right\}$, one then says that $\mathcal{F}\left[\omega^{\mathbf{a}}{ }_{a}, \xi^{\mathbf{a}}\right]$ is Fréchet differentiable at $\left\{\dot{\omega}_{a}^{\mathbf{a}}{ }_{a}, \check{\xi}^{\mathbf{a}}\right\}$. If this is the case, there exists a functional $\delta \mathcal{F}$, the Fréchet derivative, from which $\delta_{\left\{\omega_{a} \text { a }, \xi \text { a }\right\}} \mathcal{F}$ can be computed if a particular choice of the family of the variations $\left\{\delta \omega^{\mathbf{a}}{ }_{a}, \delta \xi^{\mathbf{a}}\right\}$ is considered. For more details concerning the notions of Gâteaux and Fréchet derivative and their relation, see Ref. 12.

The functional $\mathcal{F}\left[\omega^{\mathbf{a}}{ }_{a}, \xi^{\mathbf{a}}\right]$ considered in the previous paragraph depends on the coframe and components of a tensor field in terms of this basis. As the particular choice of frame involves the specification of a gauge, instead of regarding the functional $\delta \mathcal{F}$ as depending on the fields $\left[\stackrel{\circ}{\omega}_{a}^{\mathbf{a}}, \stackrel{\check{\xi}}{\xi}^{\mathbf{a}}, \delta \omega^{\mathbf{a}}{ }_{a}, \delta \xi^{\mathbf{a}}\right]$, it will be convenient to regard it as depending on $\left[\stackrel{\circ}{g}_{a b}, \stackrel{\circ}{\xi}^{a}, \delta g_{a b}, T_{a b}, \delta \xi^{a}\right]$, where the field $T_{a b}$ describes the frame gauge choice and

$$
\left.\delta g_{a b} \equiv \frac{\mathrm{d} g_{a b}}{\mathrm{~d} \lambda}\right|_{\lambda=0}
$$

where $\left\{g_{a b}\right\}$ is a family of metrics over $\mathcal{M}$ such that for fixed $\lambda$, the coframe $\stackrel{\circ}{\omega}_{a}{ }_{a}$ is $g_{a b}$-orthonormal.

Next, we consider real valued functionals depending on spinors. For concreteness consider the functional of the form $\mathcal{F}\left[g_{a b}, \epsilon^{\mathbf{A}}{ }_{A}, \kappa_{\mathbf{A}}\right]$. The Gâteaux and Fréchet derivatives of this functional are defined in the natural way by considering arbitrary families of fields $\left\{g_{a b}, \epsilon_{A}^{\mathbf{A}}, \kappa_{\mathbf{A}}\right\}$ depending on a parameter $\lambda$. The variations implied by this family of fields is then defined by

$$
\left.\delta g_{a b} \equiv \frac{\mathrm{d} g_{a b}}{\mathrm{~d} \lambda}\right|_{\lambda=0},\left.\quad \delta \epsilon_{A}^{\mathbf{A}} \equiv \frac{\mathrm{d} \epsilon^{\mathbf{A}}{ }_{A}}{\mathrm{~d} \lambda}\right|_{\lambda=0},\left.\quad \delta \kappa_{\mathbf{A}} \equiv \frac{\mathrm{d} \kappa_{\mathbf{A}}}{\mathrm{d} \lambda}\right|_{\lambda=0} .
$$

In analogy to the example considered in the previous paragraphs, it will be convenient to regard the Fréchet derivative $\delta \mathcal{F}$, which in principle depends on $\left[\stackrel{\circ}{g}_{a b},,_{\epsilon}^{\mathbf{A}}{ }_{A}, \stackrel{\circ}{\kappa}_{\mathbf{A}}, \delta g_{a b}, \delta \epsilon_{A}^{\mathbf{A}}, \delta \kappa_{\mathbf{A}}\right]$, as a functional of the arguments $\left[\stackrel{\circ}{g}_{a b}, \stackrel{\circ}{\epsilon}_{A B}, \stackrel{\circ}{\kappa}_{A}, \delta g_{a b}, \delta \kappa_{A}, T_{a b}, S_{A B}\right]$ where the field $S_{A B}$ describes the dyad gauge choice. In this way one obtains a formalism that separates the tensor frame and spin dyad gauge in the Fréchet derivatives. The main observation in the sequel is that is possible to obtain a modified variation operator $\vartheta$ which absorbs the frame and dyad gauge terms so that the Fréchet derivative depends on the parameters $\left[\stackrel{\circ}{g}_{a b}, \stackrel{\circ}{\kappa}_{A}, \delta g_{a b}, \vartheta \kappa_{A}\right]$.

Notational warning. In what follows, for ease of presentation, we mostly suppress the ring ${ }^{\circ}$ from the background quantities appearing in expressions involving variations. If an expression does not involve variations, then it holds for both the background quantities and any other one in the family. 


\section{B. Basic formulae for frames}

Consider first the expression for the metric $g_{a b}$ in terms of the coframe $\left\{\omega_{a}^{\mathbf{a}}\right\}$, namely,

$$
g_{a b}=\eta_{\mathbf{a b}} \omega^{\mathbf{a}}{ }_{a} \omega_{b}^{\mathbf{b}}{ }_{b}
$$

Applying the variational operator $\delta$ to the above expression, using the Leibnitz rule, and that $\eta_{\mathbf{a b}}$ are constants, yields

$$
\delta g_{a b}=\eta_{\mathbf{a b}} \delta \omega^{\mathbf{a}}{ }_{a} \omega^{\mathbf{b}}{ }_{b}+\eta_{\mathbf{a b}} \omega^{\mathbf{a}}{ }_{a} \delta \omega^{\mathbf{b}}{ }_{b} .
$$

In certain computations, it is useful to be able to express $\delta \omega^{\mathbf{a}}{ }_{a}$ in terms of $\delta g_{a b}$. In order to do this, it is noticed that from (5), it follows that

$$
\delta g_{a b}=2 \eta_{\mathbf{a b}} \omega_{a}^{\mathbf{a}} \delta \omega^{\mathbf{b}}{ }_{b}-2 T_{a b},
$$

where

$$
T_{a b} \equiv \eta_{\mathbf{c d}} \omega_{[a}^{\mathbf{d}} \delta \omega_{b]}^{\mathbf{c}} .
$$

It then follows that

$$
\delta\left(\omega^{\mathbf{a}}{ }_{a}\right)=\frac{1}{2} e_{\mathbf{b}}{ }^{b} \eta^{\mathbf{b a}} \delta g_{a b}-e_{\mathbf{b}}{ }^{b} \eta^{\mathbf{b a}} T_{a b} .
$$

A formula for the variation of the inverse metric can be computed by taking variations of the defining relation $\delta_{a}{ }^{b}=g_{a c} g^{c b}$. One finds that

$$
\delta\left(g^{d c}\right)=-g^{a d} g^{b c} \delta g_{a b} .
$$

A formula for the variation of the frame vectors $\left\{e_{\mathbf{a}}{ }^{a}\right\}$ in terms of the variation of $\delta \omega^{\mathbf{c}}{ }_{b}$ is obtained by computing the variation of the expression $\delta_{\mathbf{a}} \mathbf{b}=e_{\mathbf{a}}{ }^{a} \omega^{\mathbf{b}}{ }_{a}$. One finds that

$$
\delta\left(e_{\mathbf{a}}{ }^{d}\right)=-e_{\mathbf{a}}{ }^{b} e_{\mathbf{c}}{ }^{d} \delta \omega_{b}^{\mathbf{c}} .
$$

The previous expressions can be used to compute a formula for the variation of a covector $\xi_{a}$. Writing $\xi_{a}=\xi_{\mathbf{a}} \omega_{a}^{\mathbf{a}}$, one obtains that

$$
\delta \xi_{a}=\omega_{a}^{\mathbf{b}}{ }_{a} \delta\left(\xi_{\mathbf{b}}\right)+\frac{1}{2} e_{\mathbf{c}}^{b} \eta^{\mathbf{c d}} \xi_{\mathbf{d}} \delta g_{a b}-e_{\mathbf{c}}{ }^{b} \eta^{\mathbf{c d}} \xi_{\mathbf{d}} T_{a b} .
$$

Remark 4. An interpretation of the tensor $T_{a b}$ appearing in Equation (6) can be obtained by considering a situation where $\delta g_{a b}=0$. In that case, Equation (6) reduces to

$$
\delta \omega^{\mathbf{a}}{ }_{a}=-e_{\mathbf{b}}{ }^{b} \eta^{\mathbf{b a}} T_{a b} .
$$

Writing $T_{a b}=T_{\mathbf{a b}} \omega_{a}^{\mathbf{a}}{ }_{a} \omega^{\mathbf{b}}{ }_{b}$ where $T_{\mathbf{a b}}$ denote the components of $T_{a b}$ with respect to the coframe $\left\{\omega^{\mathbf{a}}{ }_{a}\right\}$, one has that

$$
\delta \omega^{\mathbf{a}}{ }_{a}=-e_{\mathbf{b}}{ }^{b} \eta^{\mathbf{b a}} T_{\mathbf{c d}} \omega_{a}^{\mathbf{c}} \omega^{\mathbf{d}}{ }_{b}=T^{\mathbf{a}} \omega^{\mathbf{c}}{ }_{a},
$$

where $T_{\mathbf{c}}^{\mathbf{a}} \equiv-\eta^{\mathbf{d a}} T_{\mathbf{c d}} \omega^{\mathbf{c}}{ }_{a}$. Comparing with the discussion in Appendix A 1, one sees that $T_{a b}$ encodes a rotation of the basis. With this observation, in what follows, we interpret the second term in Equation (6) as a gauge term.

\section{Basic formulae for spinors}

The analysis in Sec. III B admits a straightforward spinorial analogue. Given a covariant spinorial dyad $\left\{\epsilon_{A}^{\mathbf{A}}\right\}$, one can write

$$
\epsilon_{A B}=\epsilon_{\mathbf{A B}} \epsilon^{\mathbf{A}}{ }_{A} \epsilon_{B}^{\mathbf{B}}
$$

Thus, one has that

$$
\begin{aligned}
\delta \epsilon_{A B} & =\epsilon_{\mathbf{A B}} \epsilon_{B}^{\mathbf{B}} \delta \epsilon_{A}^{\mathbf{A}}+\epsilon_{\mathbf{A B}} \epsilon^{\mathbf{A}}{ }_{A} \delta \epsilon^{\mathbf{B}}{ }_{B} \\
& =2 \epsilon_{\mathbf{A B}} \epsilon_{B}^{\mathbf{B}} \delta \epsilon_{A}^{\mathbf{A}}-2 S_{A B},
\end{aligned}
$$


where

$$
S_{A B} \equiv \epsilon_{\mathbf{A B}} \epsilon_{(B}^{\mathbf{B}} \delta \epsilon^{\mathbf{A}}{ }_{A)} .
$$

The variation of the contravariant antisymmetric spinor $\epsilon^{A B}$ can be computed from the above formulae by first computing the variation of $\epsilon_{A B} \epsilon^{B C}=-\delta_{A}{ }^{C}$ and then multiplying with $\epsilon^{A D}$. We obtain that

$$
\delta\left(\epsilon^{D C}\right)=-\epsilon^{A D} \epsilon^{B C} \delta \epsilon_{A B}
$$

As, $\delta \epsilon_{A B}$ is antisymmetric we can fully express it in terms of its trace as $\delta \epsilon_{A B}=-\frac{1}{2} \epsilon_{A B} \delta \epsilon^{C}{ }_{C}$.

Now, if one wants to compute $\delta \epsilon_{A}^{\mathbf{A}}$ in terms of $\delta \epsilon_{A B}$ one has that

$$
\delta \epsilon_{A}^{\mathbf{A}}=\frac{1}{2} \epsilon^{\mathbf{A B}} \epsilon_{\mathbf{B}}{ }^{B} \delta \epsilon_{A B}+\epsilon^{\mathbf{A B}} \epsilon_{\mathbf{B}}{ }^{B} S_{A B} .
$$

If we compute the variation of $\delta_{\mathbf{A}}{ }^{\mathbf{C}}=\epsilon^{\mathbf{C}}{ }_{B} \epsilon_{\mathbf{A}}{ }^{B}$ and multiply with $\epsilon_{\mathbf{C}}{ }^{D}$ we get

$$
\delta\left(\epsilon_{\mathbf{A}}{ }^{A}\right)=-\epsilon_{\mathbf{A}}^{B} \epsilon_{\mathbf{C}}{ }^{A} \delta \epsilon^{\mathbf{C}}{ }_{B} .
$$

Now consider a covariant spinor $\phi_{A}$ and expand it with respect to the spinor dyad $\left\{\epsilon^{\mathbf{A}}{ }_{A}\right\}$ as

$$
\phi_{A}=\phi_{\mathbf{A}} \epsilon_{A}^{\mathbf{A}} \text {. }
$$

A calculation using Equation (7) yields the expression

$$
\begin{aligned}
\delta \phi_{A} & =\delta \phi_{\mathbf{A}} \epsilon^{\mathbf{A}}{ }_{A}+\phi_{\mathbf{A}} \delta \epsilon^{\mathbf{A}}{ }_{A} \\
& =\delta \phi_{\mathbf{A}} \epsilon^{\mathbf{A}}{ }_{A}+\frac{1}{2} \phi_{\mathbf{A}} \epsilon^{\mathbf{A P}} \epsilon_{\mathbf{P}}{ }^{B} \delta \epsilon_{A B}+\phi_{\mathbf{A}} \epsilon^{\mathbf{A P}} \epsilon_{\mathbf{P}}{ }^{B} S_{A B} .
\end{aligned}
$$

Using the identity $\epsilon^{\mathrm{AC}} \phi_{\mathbf{A}} \epsilon_{\mathrm{C}}^{B}=\epsilon^{C B} \phi_{C}$ the variation $\delta \phi_{A}$ can be reexpressed as

$$
\delta \phi_{A}=\left(\delta \phi_{\mathbf{A}}\right) \epsilon_{A}^{\mathbf{A}}+\frac{1}{4}\left(\delta \epsilon_{Q}^{Q}\right) \phi_{A}-S_{A}^{B} \phi_{B} .
$$

Remark 5. As in the case of Equation (6) and the tensor $T_{a b}$, the spinor $S_{A B}$ admits the interpretation of a rotation. Indeed, considering a situation where $\delta \epsilon_{A B}=0$, writing $S_{A B}=\epsilon^{\mathbf{A}}{ }_{A} \epsilon_{B}^{\mathbf{B}} S_{\mathbf{A B}}$ one finds that

$$
\begin{aligned}
\delta \epsilon^{\mathbf{A}}{ }_{A} & =\epsilon^{\mathbf{A B}} \epsilon_{\mathbf{B}}{ }^{B} S_{A B} \\
& =\epsilon^{\mathbf{A B}} \epsilon_{\mathbf{B}}{ }^{B} \epsilon^{\mathbf{P}}{ }_{A} \epsilon^{\mathbf{Q}}{ }_{B} S_{\mathbf{P Q}} \\
& =S^{\mathbf{A}}{ }_{\mathbf{B}} \epsilon^{\mathbf{B}}{ }_{A} .
\end{aligned}
$$

Comparing with Appendix A 1, we find that $S_{A B}$ encodes a rotation of the spin dyad.

\section{Variation of the soldering form}

In the reminder of this article we will consider a more general setting in which both the metric $g_{a b}$ and the antisymmetric spinor $\epsilon_{A B}$ can be varied simultaneously. To analyse the relation between the variations of these two structures it is convenient to consider the soldering form $\sigma_{a}{ }^{A A^{\prime}}$.

To compute the variation of the soldering form, one starts by computing the variation of the relation (3a). As we are treating the Infeld-van der Waerden symbols as constants, their variation vanishes - that is, although both the metric and spinor structure may vary, the formal relation between tetrads and spin dyads will be preserved. A direct combination of the methods of Sections III $\mathrm{B}$ and III $\mathrm{C}$ on formula (3a) lead, after a computation, to the expression

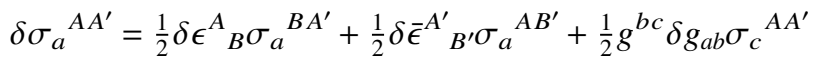

$$
\begin{aligned}
& -\bar{S}^{A^{\prime}}{ }_{B^{\prime}} \sigma_{a}{ }^{A B^{\prime}}-S^{A}{ }_{B} \sigma_{a}{ }^{B A^{\prime}}-T_{a}{ }^{b} \sigma_{b}{ }^{A A^{\prime}} .
\end{aligned}
$$

The terms in the second line of the previous expression are identified as gauge terms. Observe that in this case one has two types of gauge terms: one arising from the variation of the tensor frame and one coming from the variation of the spin frame.

If we compute the variation of Equation (4b) and multiply with $\sigma^{a}{ }_{A A^{\prime}}$ we get

$$
\delta\left(\sigma^{b}{ }_{A A^{\prime}}\right)=-\delta\left(\sigma_{a}^{B B^{\prime}}\right) \sigma^{a}{ }_{A A^{\prime}} \sigma^{b}{ }_{B B^{\prime}} .
$$


Multiplying Equation (8) with $g^{a c}{\sigma_{c}}^{B B^{\prime}}$ and splitting into irreducible parts, we get the relations

$$
\begin{aligned}
& \delta \sigma^{a}{ }_{(A}{ }^{\left(A^{\prime}\right.} \sigma_{|a| B)}{ }^{\left.B^{\prime}\right)}=\frac{1}{2} \delta g_{(A B)}{ }^{\left(A^{\prime} B^{\prime}\right)}, \\
& \delta \sigma^{a\left(A\left|B^{\prime}\right|\right.} \sigma_{a}^{B)} B^{\prime}=T^{A B}-2 S^{A B}, \\
& \delta \sigma^{a B\left(A^{\prime}\right.} \sigma_{|a| B}{ }^{\left.B^{\prime}\right)}=\bar{T}^{A^{\prime} B^{\prime}}-2 \bar{S}^{A^{\prime} B^{\prime}}, \\
& \delta \sigma^{a B B^{\prime}} \sigma_{a B B^{\prime}}=\frac{1}{2} \delta g^{B}{ }_{B}{ }^{B^{\prime}}{ }_{B^{\prime}}+\delta \epsilon^{B}{ }_{B}+\delta \bar{\epsilon}^{B^{\prime}}{ }_{B^{\prime}},
\end{aligned}
$$

where we have defined

$$
T_{A B} \equiv T_{a b} \sigma^{a}{ }_{A}^{A^{\prime}} \sigma^{b}{ }_{B A^{\prime}}, \quad \delta g_{A B A^{\prime} B^{\prime}} \equiv \delta g_{a b} \sigma^{a}{ }_{A A^{\prime}} \sigma^{b}{ }_{B B^{\prime}} .
$$

\section{E. General variations of spinors}

The formulae for the variations of the soldering form and its dual can now be used to compute the variation of arbitrary spinors under variations of the metric and spinor structures. To this end, consider spinors $\zeta^{A A^{\prime}}$ and $\xi_{A A^{\prime}}$. Making use of the Leibnitz rule one obtains the expressions

$$
\begin{aligned}
& \sigma_{a}{ }^{A A^{\prime}} \delta \zeta^{a}=\delta\left(\zeta^{A A^{\prime}}\right)-\frac{1}{4} \delta \epsilon^{B}{ }_{B} \zeta^{A A^{\prime}}-\frac{1}{4} \delta \bar{\epsilon}^{B^{\prime}{ }_{B^{\prime}}} \zeta^{A A^{\prime}}-\frac{1}{2} \delta g^{A}{ }_{B}{ }^{A^{\prime}}{ }_{B^{\prime}} \zeta^{B B^{\prime}} \\
& -\frac{1}{2} \bar{T}^{A^{\prime}}{ }_{B^{\prime}} \zeta^{A B^{\prime}}+\bar{S}^{A^{\prime}}{ }_{B^{\prime}} \zeta^{A B^{\prime}}-\frac{1}{2} T^{A}{ }_{B} \zeta^{B A^{\prime}}+S^{A}{ }_{B} \zeta^{B A^{\prime}}, \\
& \sigma^{a}{ }_{A A^{\prime}} \delta \xi_{a}=\delta\left(\xi_{A A^{\prime}}\right)+\frac{1}{4} \delta \epsilon^{B}{ }_{B} \xi_{A A^{\prime}}+\frac{1}{4} \delta \bar{\epsilon}^{B^{\prime}}{ }_{B^{\prime}} \xi_{A A^{\prime}}+\frac{1}{2} \delta g_{A}{ }^{B}{ }_{A^{\prime}}{ }^{B^{\prime}} \xi_{B B^{\prime}} \\
& +\frac{1}{2} \bar{T}_{A^{\prime}}^{B^{\prime}} \xi_{A B^{\prime}}-\bar{S}_{A^{\prime}}{ }^{B^{\prime}} \xi_{A B^{\prime}}+\frac{1}{2} T_{A}{ }^{B} \xi_{B A^{\prime}}-S_{A}{ }^{B} \xi_{B A^{\prime}},
\end{aligned}
$$

where $\zeta^{a} \equiv \sigma^{a}{ }_{B B^{\prime}} \zeta^{B B^{\prime}}$ and $\xi_{a} \equiv \sigma_{a}{ }^{B B^{\prime}} \xi_{B B^{\prime}}$. We observe that both expressions contain a combination of gauge terms involving the spinors $T_{A B}$ and $S_{A B}$.

In view of the discussion in the previous paragraph we introduce a general modified variation operator.

Definition 1. The modified variation operator $\vartheta$ is for valence 1 spinors defined by

$$
\begin{aligned}
& \vartheta \phi_{A} \equiv \delta \phi_{A}+\frac{1}{4} \delta \epsilon^{B}{ }_{B} \phi_{A}+\frac{1}{2} T_{A}^{B} \phi_{B}-S_{A}^{B} \phi_{B}, \\
& \vartheta \phi^{A} \equiv \delta \phi^{A}-\frac{1}{4} \delta \epsilon^{B}{ }_{B} \phi^{A}-\frac{1}{2} T^{A}{ }_{B} \phi^{B}+S^{A}{ }_{B} \phi^{B}, \\
& \vartheta \bar{\phi}_{A^{\prime}} \equiv \delta \bar{\phi}_{A^{\prime}}+\frac{1}{4} \delta \bar{\epsilon}^{B^{\prime}}{ }_{B^{\prime}} \bar{\phi}_{A^{\prime}}+\frac{1}{2} \bar{T}_{A^{\prime}}{ }^{B^{\prime}} \bar{\phi}_{B^{\prime}}-\bar{S}_{A^{\prime}}{ }^{B^{\prime}} \bar{\phi}_{B^{\prime}},
\end{aligned}
$$

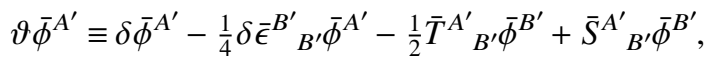

and extended to arbitrary valence spinors by the Leibnitz rule.

In particular, using the above definitions in expressions (9a) and (9b) one finds that

$$
\begin{aligned}
\sigma_{a}{ }^{A A^{\prime}} \delta \zeta^{a} & =\vartheta \zeta^{A A^{\prime}}-\frac{1}{2} \delta g^{A}{ }_{B}{ }^{A^{\prime}}{ }_{B^{\prime}} \zeta^{B B^{\prime}} \\
\sigma^{a}{ }_{A A^{\prime}} \delta \xi_{a} & =\vartheta \xi_{A A^{\prime}}+\frac{1}{2} \delta g_{A}{ }^{B}{ }_{A^{\prime}}{ }^{B^{\prime}} \xi_{B B^{\prime}}
\end{aligned}
$$

showing that $\vartheta \zeta^{A A^{\prime}}$ and $\vartheta \zeta_{A A^{\prime}}$ are frame gauge independent. Moreover, a further calculation shows that

$$
\vartheta \epsilon_{A B}=0
$$

so that the process of raising and lowering spinor indices commutes with the modified variation $\vartheta$ operator.

Remark 6. Expanding the $\phi_{A}$ in terms of the spin dyad in the $\delta \phi_{A}$ term in Definition 1 gives

$$
\vartheta\left(\phi_{A}\right)=\epsilon_{A}^{\mathbf{B}} \delta\left(\phi_{\mathbf{B}}\right)+\frac{1}{2} T_{A}^{B} \phi_{B} .
$$

Observe that the $S_{A B}$ and $\delta \epsilon_{A B}$ terms cancel out. 


\section{VARIATIONS AND THE COVARIANT DERIVATIVE}

The purpose of this section is to analyse the relation between the variation operators $\delta$ and $\vartheta$ and the Levi-Civita connection $\nabla_{a}$ of the metric $g_{a b}$.

\section{A. Basic tensorial relations}

Our analysis of the variations of expressions involving covariant derivatives is based on the following basic assumption:

Assumption. For any scalar field $f$ over $\mathcal{M}$ one has that

$$
\nabla_{a} \delta f=\delta\left(\nabla_{a} f\right)
$$

In what follows define the frame dependent tensor,

$$
\gamma_{a}{ }^{b}{ }_{c} \equiv-e_{\mathbf{c}}{ }^{b} \nabla_{a} \omega^{\mathbf{c}}{ }_{c} .
$$

The tensor $\gamma_{a}{ }^{b}{ }_{c}$ can be regarded as a convenient way of grouping the connection coefficients $\gamma_{\mathbf{a}} \mathbf{b}$ of the connection $\nabla_{a}$ with respect to the frame $\left\{e_{\mathbf{a}}{ }^{a}\right\}$. A calculation shows, indeed, that

$$
\gamma_{a}{ }^{b}{ }_{c}=\gamma_{\mathbf{a}}{ }^{\mathbf{b}} \omega^{\mathbf{a}}{ }_{a} \omega^{\mathbf{c}}{ }_{c} e_{\mathbf{b}}{ }^{b} .
$$

We can express all covariant derivatives of the cobasis and the basis in terms of $\gamma_{a}{ }^{b}{ }_{c}$ via

$$
\nabla_{a} \omega_{c}^{\mathbf{f}}=-\omega^{\mathbf{f}}{ }_{b} \gamma_{a}{ }^{b}, \quad \nabla_{d} e_{\mathbf{f}}^{b}=e_{\mathbf{f}}^{a} \gamma_{d}^{b}{ }_{a} .
$$

Differentiating the orthonormality condition $\eta^{\mathbf{a b}}=\omega_{c}^{\mathbf{a}}{ }_{c} \omega^{\mathbf{b}}{ }_{d} g^{c d}$ and multiplying with $e_{\mathbf{a}}{ }^{h} e_{\mathbf{b}}{ }^{l}$, we get the relation

$$
\gamma_{f}^{(a}{ }_{c} g^{b) c}=0
$$

encoding the metric compatibility of $\nabla_{a}$. The variation of this gives

$$
\delta \gamma_{f}^{(a b)}=\gamma_{f}^{(a|c|} \delta g^{b)}{ }_{c} .
$$

Now, for any covector $\xi_{a}$, its covariant derivative can be expanded in terms of the frame as

$$
\nabla_{a} \xi_{b}=-\omega_{d}^{\mathbf{c}} \gamma_{a}{ }^{d} \xi_{\mathbf{c}}+\omega_{b}^{\mathbf{c}} \nabla_{a} \xi_{\mathbf{c}} .
$$

Computing the variation of this last expression, and using the relations above, gives after some straightforward calculations,

$$
\begin{aligned}
\delta\left(\nabla_{a} \xi_{b}\right)= & -\delta \gamma_{a}^{c}{ }_{b} \xi_{c}+T_{c}{ }^{d} \gamma_{a b d} \xi^{c}-T_{b}{ }^{d} \gamma_{a c d} \xi^{c}+\frac{1}{2} \gamma_{a c}{ }^{d} \delta g_{b d} \xi^{c}+\frac{1}{2} \gamma_{a b}{ }^{d} \delta g_{c d} \xi^{c}+\xi^{c} \nabla_{a} T_{b c} \\
& -\frac{1}{2} \xi^{c} \nabla_{a} \delta g_{b c}+\nabla_{a} \delta \xi_{b} .
\end{aligned}
$$

In the previous calculation, Assumption (11) has been used. If we use relation (14) with $\xi_{a}=\nabla_{a} f$, antisymmetrize over $a$ and $b$, and assume that the connection is torsion free, we get

$$
0=\left(T_{c}{ }^{d} \gamma_{[a b] d}+\frac{1}{2} \delta g_{c}{ }^{d} \gamma_{[a b] d}-\delta \gamma_{[a|c| b]}+\nabla_{[a} T_{b] c}-\frac{1}{2} \nabla_{[a} \delta g_{b] c}+T_{[a}{ }^{d} \gamma_{b] c d}+\frac{1}{2} \gamma_{[a|c|}{ }^{d} \delta g_{b] d}\right) \nabla^{c} f .
$$

Hence, the torsion free condition is encoded by

$$
\delta \gamma_{[a|c| b]}=T_{c}{ }^{d} \gamma_{[a b] d}+\frac{1}{2} \delta g_{c}{ }^{d} \gamma_{[a b] d}+\nabla_{[a} T_{b] c}-\frac{1}{2} \nabla_{[a} \delta g_{b] c}+T_{[a}{ }^{d} \gamma_{b] c d}+\frac{1}{2} \gamma_{[a|c|}{ }^{d} \delta g_{b] d} .
$$

Now, using the identity

$$
\delta \gamma_{a b c}=\delta \gamma_{[a|b| c]}-\delta \gamma_{[a|c| b]}+\delta \gamma_{[b|a| c]}+\delta \gamma_{a(b c)}-\delta \gamma_{b(a c)}+\delta \gamma_{c(a b)},
$$

we can use Equations (13) and (15) to compute

$$
\delta \gamma_{a b c}=-T_{c}{ }^{d} \gamma_{a b d}+T_{b}{ }^{d} \gamma_{a c d}+\frac{1}{2} \gamma_{a c}{ }^{d} \delta g_{b d}+\frac{1}{2} \gamma_{a b}{ }^{d} \delta g_{c d}-\nabla_{a} T_{b c}-\frac{1}{2} \nabla_{b} \delta g_{a c}+\frac{1}{2} \nabla_{c} \delta g_{a b} .
$$

It follows then that Equation (14) can therefore be simplified to

$$
\delta\left(\nabla_{a} \xi_{b}\right)=\nabla_{a}\left(\delta \xi_{b}\right)-\frac{1}{2} g^{c d}\left(\nabla_{a} \delta g_{b c}+\nabla_{b} \delta g_{a c}-\nabla_{c} \delta g_{a b}\right) \xi_{d}
$$


It is important to observe that this formula is a tensorial expression. Hence, it allows to define a transition tensor

$$
Q_{b}{ }^{a}{ }_{c} \equiv \frac{1}{2} g^{a d}\left(\nabla_{b} \delta g_{d c}+\nabla_{c} \delta g_{b d}-\nabla_{d} \delta g_{b c}\right)
$$

relating the connections $\nabla_{a}$ and $\delta \nabla_{a}$. This is not surprising as it is well known that the space of covariant derivatives on a manifold is an affine space. Making use of the definition of $Q_{b}{ }^{a}{ }_{c}$, Equation (17) takes the suggestive form

$$
\delta\left(\nabla_{a} \xi_{b}\right)=\nabla_{a}\left(\delta \xi_{b}\right)-Q_{b}{ }_{a}{ }_{a} \xi_{d} .
$$

Furthermore, making use of the Leibnitz rule one finds that for an arbitrary vector $v^{a}$ one has

$$
\delta\left(\nabla_{a} v^{b}\right)=\nabla_{a}\left(\delta v^{b}\right)+Q_{c}{ }^{b} v^{c} .
$$

The extension to higher valence tensors follows in a similar manner.

\section{B. Spinorial expressions}

In order to discuss the variations of the spinor covariant derivative $\nabla_{A A^{\prime}}$ associated to the LeviCivita connection $\nabla_{a}$ it is convenient to define a spinorial analogue of the tensor $\gamma_{a}{ }^{b}{ }_{c}$-namely

$$
\gamma_{a}{ }^{B}{ }_{C} \equiv-\epsilon_{\mathbf{C}}{ }^{B} \nabla_{a} \epsilon^{\mathbf{C}} \text {. }
$$

The hybrid $\gamma_{a}{ }^{B} C$ is related to $\gamma_{a}{ }^{B B^{\prime}} C C^{\prime} \equiv \gamma_{a}{ }^{b}{ }_{c} \sigma_{b}{ }^{B B^{\prime}} \sigma^{c}{ }_{C C^{\prime}}$ through the decomposition

$$
\gamma_{a}{ }^{B B^{\prime}}{ }_{C C^{\prime}}=\gamma_{a}{ }^{B}{ }_{C} \delta_{C^{\prime}}{ }^{B^{\prime}}+\bar{\gamma}_{a}{ }^{B^{\prime}}{ }_{C^{\prime}} \delta_{C}{ }^{B} \text {. }
$$

It follows then that

$$
\gamma_{a}{ }^{B} C=\frac{1}{2} \gamma_{a}{ }^{c}{ }^{b}{ }^{b}{ }_{C B^{\prime}} \sigma_{c}{ }^{B B^{\prime}} .
$$

From this last expression, it can then be verified that

$$
\gamma_{a B C}=\gamma_{a C B} .
$$

The variational derivative of $\gamma_{a}{ }^{B} C$ can be computed using Equation (20). One finds that

$$
\begin{aligned}
& \delta\left(\gamma_{a}{ }^{B}{ }_{C}\right)=\frac{1}{4} Q_{a c d} \sigma^{d B B^{\prime}} \sigma^{c}{ }_{C B^{\prime}}-\frac{1}{4} Q_{a c d} \sigma^{d B B^{\prime}} \sigma^{c}{ }_{C B^{\prime}}-\frac{1}{2} \gamma_{a c d} S_{C D} \sigma^{c B B^{\prime}} \sigma^{d D}{ }_{B^{\prime}} \\
& -\frac{1}{2} \gamma_{a c d} S^{B}{ }_{D} \sigma^{c}{ }_{C}^{B^{\prime}} \sigma^{d D}{ }_{B^{\prime}}-\frac{1}{2} \nabla_{a} T_{C}^{B} \text {. }
\end{aligned}
$$

In this last expression observe, in particular, the appearance of the gauge spinors $S_{A B}$ and $T_{A B}$. In turn, Equation (21) can be used to compute the variation of the covariant derivative of an arbitrary spinor $\kappa_{A}$. Expanding $\kappa_{A}$ in terms of the spin dyad and differentiating, we get

$$
\nabla_{a} \kappa_{B}=\epsilon^{\mathrm{C}}{ }_{B} \nabla_{a} \kappa_{\mathbf{C}}-\gamma_{a}{ }^{C}{ }_{B} K_{C} .
$$

It follows that the variation of this last expression is given by

$$
\begin{aligned}
\delta\left(\nabla_{a} \kappa_{A}\right)= & \nabla_{a} \delta \kappa_{A}-\frac{1}{2} \kappa^{B} \nabla_{a} T_{A B}+\kappa^{B} \nabla_{a} S_{A B}+\frac{1}{4} \kappa_{A} \nabla_{a} \delta \epsilon^{B}{ }_{B} \\
& +\frac{1}{4} Q_{a b c} \kappa^{B} \sigma^{b}{ }_{A} A^{\prime} \sigma^{c}{ }_{B A^{\prime}}-\frac{1}{4} Q_{a c b} \kappa^{B} \sigma^{b}{ }_{A} A^{\prime} \sigma^{c}{ }_{B A^{\prime}} \\
= & \nabla_{a} \vartheta \kappa_{A}-\frac{1}{4} \delta \epsilon^{B}{ }_{B} \nabla_{a} \kappa_{A}+\frac{1}{2} T_{A B} \nabla_{a} \kappa^{B}-S_{A B} \nabla_{a} \kappa^{B} \\
& +\frac{1}{4} Q_{a b c} \kappa^{B} \sigma^{b}{ }_{A} A^{\prime} \sigma^{c}{ }_{B A^{\prime}}-\frac{1}{4} Q_{a c b} \kappa^{B} \sigma^{b}{ }_{A} A^{\prime} \sigma^{c}{ }_{B A^{\prime}} .
\end{aligned}
$$

In order to write the spinorial derivative $\nabla_{A A^{\prime} \kappa_{B}}$ (rather than $\nabla_{a} \kappa_{B}$ ), it is convenient to define the spinor,

$$
Q_{A A^{\prime} B C} \equiv-\frac{1}{2} \sigma^{a}{ }_{A A^{\prime}} \sigma^{b}{ }_{B}{ }^{B^{\prime}} \sigma^{c}{ }_{C B^{\prime}} Q_{[b c] a} .
$$

Theorem 1. The variation of the covariant derivative of a spinor is given by

$$
\begin{aligned}
& \vartheta\left(\nabla_{A A^{\prime} K_{B}}\right)=\nabla_{A A^{\prime \prime}} \vartheta \kappa_{B}+9_{A A^{\prime} B C} K^{C}-\frac{1}{2} \delta g_{A C A^{\prime} B^{\prime}} \nabla^{C B^{\prime}} \kappa_{B}, \\
& \vartheta\left(\nabla_{A A^{\prime}} \bar{\kappa}_{B^{\prime}}\right)=\nabla_{A A^{\prime}} \vartheta \bar{\kappa}_{B^{\prime}}+\bar{Q}_{A^{\prime} A B^{\prime} C^{\prime}} \bar{K}^{C^{\prime}}-\frac{1}{2} \delta g_{A B A^{\prime} C^{\prime}} \nabla^{B C^{\prime}} \bar{\kappa}_{B^{\prime}} .
\end{aligned}
$$


Proof. Using the expressions in the previous paragraphs, one has that

$$
\begin{aligned}
& \delta\left(\nabla_{A A^{\prime} K_{B}}\right)=\nabla_{A A^{\prime}} \vartheta \kappa_{B}+9_{A A^{\prime} B C} K^{C}-\frac{1}{4} \delta \epsilon_{C}^{C} \nabla_{A A^{\prime} K_{B}}+\frac{1}{2} T_{B C} \nabla_{A A^{\prime} K^{C}}-S_{B C} \nabla_{A A^{\prime} K^{C}} \\
& -\delta \sigma_{a}{ }^{C B^{\prime}} \sigma^{a}{ }_{A A^{\prime}} \nabla_{C B^{\prime} K_{B}} \\
& =\nabla_{A A^{\prime}} \vartheta \kappa_{B}+{ }_{A A^{\prime} B C} K^{C}-\frac{1}{2} \delta \epsilon^{C}{ }_{C} \nabla_{A A^{\prime} K_{B}}-\frac{1}{4} \delta \bar{\epsilon}^{B^{\prime}}{ }_{B^{\prime}} \nabla_{A A^{\prime} K_{B}}+\frac{1}{2} T_{B C} \nabla_{A A^{\prime} K^{C}} \\
& -S_{B C} \nabla_{A A^{\prime} K^{C}}+\frac{1}{2} \bar{T}_{A^{\prime} B^{\prime}} \nabla_{A}{ }^{B^{\prime}} \kappa_{B}-\bar{S}_{A^{\prime} B^{\prime}} \nabla_{A}{ }^{B^{\prime}} \kappa_{B}+\frac{1}{2} T_{A C} \nabla^{C}{ }_{A^{\prime} K_{B}} \\
& -S_{A C} \nabla_{A^{\prime} K_{B}}-\frac{1}{2} \delta g_{A C A^{\prime} B^{\prime}} \nabla^{C B^{\prime}} K_{B} \text {. }
\end{aligned}
$$

Expressing the above formula in terms of the modified variation $\vartheta$, we get (23a). Equation (23b) is given by complex conjugation.

\section{Decomposition of ${ }_{A A A^{\prime} B C}$}

Starting from the definition in Equation (22), a calculation yields

$$
\begin{aligned}
Q_{A A^{\prime} B C} & =-\frac{1}{4} \sigma^{a}{ }_{A A^{\prime}} \sigma^{b}{ }_{B}{ }^{B^{\prime}} \sigma^{c}{ }_{C B^{\prime}} \nabla_{b} \delta g_{a c}+\frac{1}{4} \sigma_{A A^{\prime}}^{a} \sigma^{b}{ }_{B}{ }^{B^{\prime}} \sigma^{c}{ }_{C B^{\prime}} \nabla_{c} \delta g_{a b} \\
& =-\frac{1}{2} \nabla_{(B}{ }^{B^{\prime}} \delta g_{C) A B^{\prime} A^{\prime} .}
\end{aligned}
$$

The above expression can be conveniently decomposed in irreducible terms. To this end, one defines

$$
G \equiv \delta g_{C}{ }_{C}^{C^{\prime}}{ }_{C^{\prime}}, \quad G_{A B A^{\prime} B^{\prime}} \equiv \delta g_{(A B)\left(A^{\prime} B^{\prime}\right)} .
$$

If we also decompose $Q_{A B C A^{\prime}}$ into irreducible parts, we get

$$
9_{A A^{\prime} B C}=-\frac{1}{2} \nabla_{(A} B^{\prime} G_{B C) A^{\prime} B^{\prime}}+\frac{1}{8} \epsilon_{A(B} \nabla_{C) A^{\prime}} G-\frac{1}{6} \epsilon_{A(B} \nabla^{D B^{\prime}} G_{C) D A^{\prime} B^{\prime}} .
$$

For future use, we notice the following relations which follow from the decomposition in irreducible components of Equation (24) and the reality of $\delta g_{A B A^{\prime} B^{\prime}}$ :

$$
\begin{aligned}
{ }^{B}{ }_{A^{\prime} A B} & =-\frac{3}{16} \nabla_{A A^{\prime}} G+\frac{1}{4} \nabla_{B B^{\prime}} G_{A^{\prime}}{ }_{A^{\prime}}^{B^{\prime},} \\
\bar{\varphi}^{B^{\prime}}{ }_{A A^{\prime} B^{\prime}} & ={ }^{B}{ }_{A^{\prime} A B}, \\
\nabla_{B B^{\prime}} G_{C D A^{\prime}}{ }^{B^{\prime}} & =2{ }_{B A^{\prime} C D}-4 \varphi^{A}{ }_{A^{\prime}(C|A|} \epsilon_{D) B}-\frac{1}{2} \epsilon_{(C|B|} \nabla_{D) A^{\prime}} G, \\
\nabla_{B A^{\prime}} G_{A}{ }^{B}{ }_{B^{\prime} C^{\prime}} & =2 \bar{Q}_{A^{\prime} A B^{\prime} C^{\prime}}-4 \varphi^{B}{ }_{\left(B^{\prime}|A B|\right.} \bar{\epsilon}_{\left.C^{\prime}\right) A^{\prime}}-\frac{1}{2} \bar{\epsilon}_{\left(B^{\prime} \mid A^{\prime}\right.} \nabla_{\left.A \mid C^{\prime}\right)} G .
\end{aligned}
$$

We also define the field

$$
\begin{aligned}
F^{A A^{\prime}} & \equiv \nabla_{B B^{\prime}} \delta g^{A B A^{\prime} B^{\prime}}-\frac{1}{2} \nabla^{A A^{\prime}} \delta g^{B}{ }_{B} B^{B^{\prime}}{ }^{\prime} \\
& =\nabla_{B B^{\prime}} G^{A B A^{\prime} B^{\prime}}-\frac{1}{4} \nabla^{A A^{\prime}} G .
\end{aligned}
$$

In Sec. IV C, we will see that this can be interpreted as a gauge source function for the linearised diffeomorphisms.

\section{Diffeomorphism dependence}

We will now briefly consider the dependence on diffeomorphisms. Let $\phi_{\lambda}$ be a one parameter group of diffeomorphisms generated by a vector field $\xi^{a}$ and such that $g_{a b}[\lambda]=\phi_{-\lambda}^{*} \stackrel{\circ}{g}_{a b}$. The metrics in this family have the same geometric content and one readily finds that

$$
\delta g_{a b}=\mathcal{L}_{\xi} g_{a b}=2 \nabla_{(a} \xi_{b)} .
$$

Moreover, a further computation yields

$$
\begin{aligned}
Q_{A A^{\prime} B C} & =-\frac{1}{2} \nabla_{(C}{ }^{B^{\prime}} \nabla_{B) B^{\prime}} \xi_{A A^{\prime}}-\frac{1}{2} \nabla_{(C}{ }^{B^{\prime}} \nabla_{\left|A A^{\prime}\right|} \xi_{B) B^{\prime}} \\
F^{A A^{\prime}} & =\nabla_{B B^{\prime}} \nabla^{B B^{\prime}} \xi^{A A^{\prime}}-6 \Lambda \xi^{A A^{\prime}}+2 \Phi^{A}{ }_{B}{ }^{A^{\prime}}{ }_{B^{\prime}} \xi^{B B^{\prime}}
\end{aligned}
$$


Given a general family of metrics $g_{a b}[\lambda]$, we can compute the field $F^{A A^{\prime}}$ associated to the family. Given any $\widetilde{F}^{A A^{\prime}}$, we can then solve the wave equation

$$
\widetilde{F}^{A A^{\prime}}-F^{A A^{\prime}}=-6 \Lambda \xi^{A A^{\prime}}+2 \Phi_{B}^{A}{ }_{B}^{A^{\prime}}{ }_{B^{\prime}} \xi^{B B^{\prime}}+\nabla_{B B^{\prime}} \nabla^{B B^{\prime}} \xi^{A A^{\prime}} .
$$

The solution $\xi^{a}=\sigma_{A A^{\prime}}^{a} \xi^{A A^{\prime}}$ to this equations will then give a one parameter group of diffeomorphisms $\phi_{\lambda}$, such that $g_{a b}[\lambda]=\phi_{-\lambda}^{*} g_{a b}[\lambda]$ has the same geometric content, but with corresponding $\widetilde{F}^{A A^{\prime}}$. With this observation, we can interpret (25) as a gauge source function for the linearised diffeomorphisms.

\section{VARIATION OF CURVATURE}

The purpose of this section is to compute the variation of the various spinorial components of the curvature tensor. As it will be seen below, the starting point of this computation is the commutator of covariant derivatives.

We start by computing the variation of

$$
\left.\square_{(A B} K_{C}\right)=\nabla_{(A}^{A^{\prime}} \nabla_{\left.B\left|A^{\prime}\right| K_{C}\right)}=-\Psi_{A B C D} K^{D}
$$

for an arbitrary spinor $\kappa_{A}$. A direct calculation using the Leibnitz rule for the modified operator $\vartheta$ gives

$$
\begin{aligned}
& \left.\left.\Psi_{A B C D} \vartheta K^{D}+\vartheta\left(\Psi_{A B C D}\right) K^{D}=-\nabla_{(A} A^{\prime} \nabla_{B\left|A^{\prime}\right|} \vartheta \kappa_{C}\right)+\frac{1}{4} G \nabla_{(A} A^{\prime} \nabla_{B\left|A^{\prime}\right|} K_{C}\right) \\
& -\frac{1}{2} G_{(A}{ }^{D A^{\prime} B^{\prime}} \nabla_{B \mid A^{\prime}} \nabla_{\left.D B^{\prime} \mid K_{C}\right)}+\frac{1}{2} G_{(A} D A^{\prime} B^{\prime} \nabla_{\left|D A^{\prime}\right|} \nabla_{\left.B\left|B^{\prime}\right| K_{C}\right)} \\
& +Q_{(A} A_{B}{ }^{D} \nabla_{\left.\left|D A^{\prime}\right| K_{C}\right)}+\bar{\rho}^{A^{\prime}}{ }_{\left(A\left|A^{\prime}\right|\right.}^{B^{\prime}} \nabla_{\left.B\left|B^{\prime}\right| K_{C}\right)} \\
& -K^{D} \nabla_{(A} A^{A^{\prime}} Q_{\left.B\left|A^{\prime}\right| C\right) D}+\frac{1}{8} \nabla_{(A} A^{\prime} G \nabla_{\left.B\left|A^{\prime}\right| K_{C}\right)} \\
& +\frac{1}{2} \nabla_{(A}{ }^{A^{\prime}} G_{B}{ }^{D}{ }_{\mid A^{\prime}}{ }^{\prime} \nabla_{D B^{\prime} \mid} K_{C)} \\
& =\Psi_{A B C D} \vartheta \kappa^{D}-\frac{1}{4} G \Psi_{A B C D} K^{D}-\kappa^{D} \nabla_{(A} A^{\prime} Q_{\left.B\left|A^{\prime}\right| C\right) D} \\
& +\frac{1}{2} \kappa^{D} G_{(A B}{ }^{A^{\prime} B^{\prime}} \Phi_{C) D A^{\prime} B^{\prime}}
\end{aligned}
$$

The above expression holds for all $\kappa^{A}$, and therefore we can conclude that

$$
\vartheta\left(\Psi_{A B C D}\right)=-\frac{1}{4} G \Psi_{A B C D}-\nabla_{(A}{ }^{A^{\prime}}{ }_{Q_{\left.B\left|A^{\prime}\right| C\right) D}}+\frac{1}{2} G_{(A B}{ }^{A^{\prime} B^{\prime}} \Phi_{C) D A^{\prime} B^{\prime}} .
$$

The symmetry of $\Psi_{A B C D}$ can be used to simplify this last expression- the trace of the right hand side can be shown to vanish due to the commutators.

If we compute the variation of

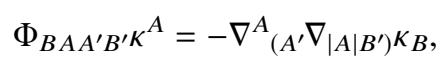

we get

$$
\begin{aligned}
& \Phi_{B A A^{\prime} B^{\prime}} \vartheta \kappa^{A}+\vartheta\left(\Phi_{B A A^{\prime} B^{\prime}}\right) K^{A}=-\nabla_{\left(A^{\prime}\right.}^{A} \nabla_{\left.|A| B^{\prime}\right)} \vartheta \kappa_{B}+\frac{1}{4} G \nabla_{\left(A^{\prime}\right.}^{A} \nabla_{\left.|A| B^{\prime}\right)} K_{B} \\
& -\frac{1}{2} G^{A C}{ }_{\left(A^{\prime}\right.} C^{\prime} \nabla_{\left.|A| B^{\prime}\right)} \nabla_{C C^{\prime} K_{B}}+\frac{1}{2} G^{A C}{ }_{\left(A^{\prime}\right.} C^{\prime} \nabla_{\mid A C^{\prime}} \nabla_{\left.C \mid B^{\prime}\right)} K_{B}
\end{aligned}
$$

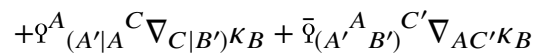

$$
\begin{aligned}
& -K^{A} \nabla^{C}{ }_{\left(A^{\prime},{ }^{\prime}|C| B^{\prime}\right) B A}+\frac{1}{8} \nabla^{A}{ }_{\left(A^{\prime}\right.} G \nabla_{\left.|A| B^{\prime}\right)} K_{B} \\
& +\frac{1}{2} \nabla^{A}{ }_{\left(A^{\prime}\right.} G_{|A|}{ }^{C}{ }_{\left.B^{\prime}\right)}{ }^{\prime} \nabla_{C C^{\prime} K_{B}} \\
& =\Phi_{B A A^{\prime} B^{\prime}} \vartheta \kappa^{A}+G_{B A A^{\prime} B^{\prime}} \Lambda \kappa^{A}-\frac{1}{4} G \Phi_{B A A^{\prime} B^{\prime}} K^{A} \\
& +\frac{1}{2} G^{C D}{ }_{A^{\prime} B^{\prime}} \Psi_{B A C D} K^{A}-K^{A} \nabla^{C}{ }_{\left(A^{\prime}|C| B^{\prime}\right) B A} .
\end{aligned}
$$

The last relation holds for all $\kappa^{A}$, and therefore we can obtain an expression for $\vartheta \Phi_{A B A^{\prime} B^{\prime}}$. 
Now, using the definition of $9_{A B C A^{\prime}}$, commuting derivatives, and exploiting the irreducible decomposition of the various fields involved, one gets

$$
\begin{aligned}
& \nabla_{A A^{\prime}{ }^{O} C A^{\prime}}{ }_{B C}=-\frac{1}{2} G_{B} C A^{\prime} B^{\prime} \Phi_{A C A^{\prime} B^{\prime}}-\frac{1}{2} G_{A} C A^{\prime} B^{\prime} \Phi_{B C A^{\prime} B^{\prime}}
\end{aligned}
$$

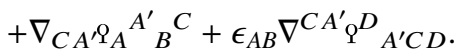

If we compute the variation of

$$
\Lambda \kappa_{A}=\frac{1}{3} \nabla_{(A}{ }^{A^{\prime}} \nabla_{B) A^{\prime} K^{B},}
$$

we get, after a lengthy computation, that

$$
\begin{aligned}
& \Lambda \vartheta \kappa_{A}+\vartheta(\Lambda) \kappa_{A}=\frac{1}{6} \kappa^{B} \nabla_{A A^{\prime} q^{C A^{\prime}}}{ }_{B C}-\frac{1}{6} \nabla_{A A^{\prime}} \nabla_{B}{ }^{A^{\prime}} \vartheta \kappa^{B}+\frac{1}{24} G \nabla_{A A^{\prime}} \nabla_{B}{ }^{A^{\prime}} K^{B} \\
& +\frac{1}{6} \bar{Q}^{B^{\prime}}{ }_{B A^{\prime} B^{\prime}} \nabla_{A}{ }^{A^{\prime}} K^{B}-\frac{1}{12} G_{B C A^{\prime} B^{\prime}} \nabla_{A}{ }^{B^{\prime}} \nabla^{C A^{\prime}} K^{B}-\frac{1}{48} \nabla_{A}{ }^{A^{\prime}} G \nabla_{B A^{\prime} K^{B}} \\
& -\frac{1}{6} \nabla_{B A^{\prime}} \nabla_{A} A^{\prime} \vartheta K^{B}+\frac{1}{24} G \nabla_{B A^{\prime}} \nabla_{A} A^{\prime} K^{B}+\frac{1}{6} \bar{Q}^{B^{\prime}}{ }_{A A^{\prime} B^{\prime}} \nabla_{B} A^{A^{\prime}} K^{B}
\end{aligned}
$$

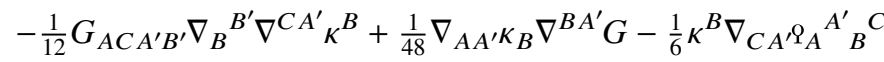

$$
\begin{aligned}
& -\frac{1}{6} Q_{A A^{\prime} B C} \nabla^{C A^{\prime}} K^{B}-\frac{1}{6} Q_{B A^{\prime} A C} \nabla^{C A^{\prime}} K^{B}+\frac{1}{12} \nabla_{A B^{\prime}} G_{B C A^{\prime}}{ }^{B^{\prime}} \nabla^{C A^{\prime}} K^{B} \\
& +\frac{1}{12} \nabla_{B B^{\prime}} G_{A C A^{\prime}}{ }^{B^{\prime}} \nabla^{C A^{\prime}} K^{B}+\frac{1}{12} G_{B C A^{\prime} B^{\prime}} \nabla^{C B^{\prime}} \nabla_{A}{ }^{A^{\prime}} K^{B} \\
& +\frac{1}{12} G_{A C A^{\prime} B^{\prime}} \nabla^{C B^{\prime}} \nabla_{B}{ }^{A^{\prime}} K^{B}
\end{aligned}
$$

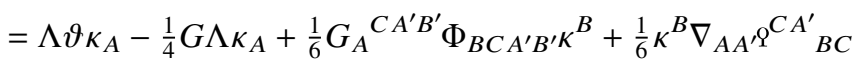

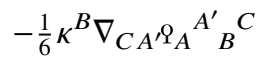

$$
\begin{aligned}
& =\Lambda \vartheta \kappa_{A}-\frac{1}{4} G \Lambda \kappa_{A}+\frac{1}{12} G^{B C A^{\prime} B^{\prime}} \Phi_{B C A^{\prime} B^{\prime} K_{A}}-\frac{1}{6} \kappa_{A} \nabla_{C A^{\prime} \mathrm{P} B A^{\prime}{ }_{B}{ }^{C}} \text {. }
\end{aligned}
$$

In the last equality, we have used relation (27) and the irreducible decomposition of $G_{A} C A^{\prime} B^{\prime}$ $\Phi_{B C A^{\prime} B^{\prime}}$. From here, we can deduce an expression for $\vartheta \Lambda$.

We summarise the discussion of this section in the following:

Theorem 2. The modified variation of the curvature spinors is given by

$$
\begin{aligned}
\vartheta \Psi_{A B C D} & =-\frac{1}{4} G \Psi_{A B C D}-\nabla_{(A} A^{\prime} Q_{\left.B\left|A^{\prime}\right| C D\right)}+\frac{1}{2} G_{(A B}{ }^{A^{\prime} B^{\prime}} \Phi_{C D) A^{\prime} B^{\prime}}, \\
\vartheta \Phi_{A B A^{\prime} B^{\prime}} & =G_{A B A^{\prime} B^{\prime}} \Lambda-\frac{1}{4} G \Phi_{A B A^{\prime} B^{\prime}}+\frac{1}{2} G^{C D}{ }_{A^{\prime} B^{\prime}} \Psi_{A B C D}-\nabla^{C}{ }_{\left(A^{\prime}|C| B^{\prime}\right) A B}, \\
\vartheta \Lambda & =-\frac{1}{4} G \Lambda+\frac{1}{12} G^{B C A^{\prime} B^{\prime}} \Phi_{B C A^{\prime} B^{\prime}}-\frac{1}{6} \nabla_{C A^{\prime} Q B A^{\prime}{ }_{B} C} .
\end{aligned}
$$

Remark 7. For a pure gauge transformation (26), we get after a lengthy but straightforward calculation using commutators that

$$
\begin{aligned}
& \vartheta(\Lambda)=\left(\mathcal{L}_{\xi} \Lambda\right),
\end{aligned}
$$

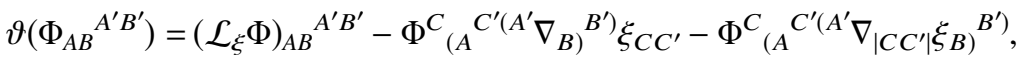

$$
\begin{aligned}
& \vartheta\left(\Psi_{A B C D}\right)=\left(\mathcal{L}_{\xi} \Psi\right)_{A B C D}-\Psi_{A B C D} \nabla^{F A^{\prime}} \xi_{F A^{\prime}},
\end{aligned}
$$

where $^{11}$

$$
\begin{aligned}
& \left(\mathcal{L}_{\xi} \Phi\right)_{A B}{ }^{A^{\prime} B^{\prime}} \equiv \xi^{C C^{\prime}} \nabla_{C C^{\prime}} \Phi_{A B}{ }^{A^{\prime} B^{\prime}}+2 \Phi^{C}{ }_{(A} C^{\prime}\left(A^{\prime} \nabla_{B}\right)^{\left.B^{\prime}\right)} \xi_{C C^{\prime}}, \\
& \left(\mathcal{L}_{\xi} \Psi\right)_{A B C D} \equiv \xi^{F A^{\prime}} \nabla_{F A^{\prime}} \Psi_{A B C D}+2 \Psi_{(A B C}{ }^{F} \nabla_{D)}{ }^{A^{\prime}} \xi_{F A^{\prime}} .
\end{aligned}
$$

In this last calculation, we have used the Bianchi identity in the form

$$
\nabla_{A^{\prime}}^{D} \Psi_{A B C D}=\nabla_{(A}{ }^{B^{\prime}} \Phi_{B) C A^{\prime} B^{\prime}}+\epsilon_{C(A} \nabla_{B) A^{\prime}} \Lambda
$$




\section{VARIATIONS OF SPACE-SPINOR EXPRESSIONS}

The analysis of Sections III-V can be adapted to consider variations of spinorial fields in a space-spinor formalism. This formalism can be used to analyse variational problems in 3dimensional Riemannian manifolds.

\section{A. Basic formalism}

In what follows, let $\left(\mathcal{S}, h_{i j}\right)$ denote a 3-dimensional Riemannian manifold with negative-definite metric. On $\left(\mathcal{S}, h_{i j}\right)$, we assume the existence of a spinor structure with an antisymmetric spinor $\epsilon_{A B}$. In addition, we assume that the spinor structure is endowed with an Hermitian product. It follows from this assumption that there exists an Hermitian spinor $\varpi_{A A^{\prime}}$ such that given two spinors $\xi_{A}$ and $\eta_{B}$ the Hermitian inner product can be expressed as

$$
\xi_{A} \hat{\eta}^{A} \equiv \varpi_{A A^{\prime}} \bar{\eta}^{A^{\prime}} \xi^{A}
$$

The spinor $\hat{\eta}^{A}$ defined by the above relation is called the Hermitian conjugate of $\eta^{A}$.

Let $e_{\mathbf{k}}{ }^{l}, \omega^{\mathbf{k}}{ }_{l}$ denote, respectively, an orthonormal frame and coframe of $\left(\mathcal{S}, h_{i j}\right)$ and let $\epsilon^{\mathbf{A}}{ }_{B}$ denote a normalised spin dyad such that the components of $\epsilon_{A B}$ and $\varpi_{A A^{\prime}}$ are given, respectively, by

$$
\epsilon_{\mathrm{AB}}=\left(\begin{array}{cc}
0 & 1 \\
-1 & 0
\end{array}\right), \quad \varpi_{\mathbf{A A}^{\prime}}=\left(\begin{array}{cc}
1 & 0 \\
0 & 1
\end{array}\right)
$$

The transformations of the spin dyad respecting the above expressions is given by $S U(2, \mathbb{C})$ matrices $O_{\mathrm{A}}{ }^{\mathrm{B}}$.

The correspondence between spatial tensors and spinors is realised by the spatial Infeld-van der Waerden symbols $\sigma_{\mathbf{k}}{ }^{\mathbf{A B}}$ and $\sigma^{\mathbf{k}} \mathbf{A B}$. Given an arbitrary $v^{k} \in T \mathcal{S}$ and $\beta_{k} \in T^{*} \mathcal{S}$, one has that

$$
v^{\mathbf{k}} \mapsto v^{\mathbf{A B}}=v^{\mathbf{k}}{\sigma_{\mathbf{k}}}^{\mathbf{A B}}, \quad \beta_{\mathbf{k}} \mapsto \beta_{\mathbf{A B}}=\beta_{\mathbf{k}} \sigma^{\mathbf{k}}{ }_{\mathbf{A B}},
$$

where

$$
v^{\mathbf{k}} \equiv v^{k} \omega_{k}^{\mathbf{k}}, \quad \beta_{\mathbf{k}} \equiv \beta_{k} e_{\mathbf{k}}^{k} .
$$

In more explicit terms, the correspondence is

$$
\left(v^{1}, v^{2}, v^{3}\right) \mapsto \frac{1}{\sqrt{2}}\left(\begin{array}{cc}
-v^{1}-\mathrm{i} v^{2} & v^{3} \\
v^{3} & v^{1}-\mathrm{i} v^{2}
\end{array}\right), \quad\left(\beta_{1}, \beta_{2}, \beta_{3}\right) \mapsto \frac{1}{\sqrt{2}}\left(\begin{array}{cc}
-\beta_{1}+\mathrm{i} \beta_{2} & \beta_{3} \\
\beta_{3} & \beta_{1}+\mathrm{i} \beta_{2}
\end{array}\right) .
$$

From these, we define the spatial soldering form to be

$$
\begin{aligned}
\sigma_{k}{ }^{A B} & \equiv \omega_{k}^{\mathbf{l}} \epsilon_{\mathbf{C}}{ }^{A} \epsilon_{\mathbf{D}}{ }^{B} \sigma_{\mathbf{l}}{ }^{\mathbf{C D}}, \\
\sigma^{k}{ }_{A B} & \equiv \epsilon^{\mathbf{C}}{ }_{A} \epsilon^{\mathbf{D}}{ }_{B} e_{\mathbf{l}}^{k} \sigma^{\mathbf{l}} \mathbf{C D} .
\end{aligned}
$$

As we allow the spinor and tensor frames to be independent, the soldering form will therefore be frame dependent. However, we will always have the universal relations

$$
\begin{aligned}
\sigma_{k}{ }^{C D} \sigma^{l}{ }_{C D} & =\delta_{k}{ }^{l}, \\
h_{k l} & =\sigma_{k}{ }^{A B} \sigma_{l}{ }^{C D} \epsilon_{C A} \epsilon_{D B} .
\end{aligned}
$$

The Hermitian conjugate of

$$
\phi_{A}=\phi_{0} \epsilon_{A}^{0}+\phi_{1} \epsilon_{A}^{1}
$$

is given by

$$
\hat{\phi}_{A}=-\bar{\phi}_{\mathbf{1}^{\prime}} \epsilon_{A}^{\mathbf{0}}+\bar{\phi}_{\mathbf{0}^{\prime}} \epsilon^{\mathbf{1}}{ }_{A} .
$$

It clearly follows that

$$
\hat{\hat{\phi}}_{A}=-\phi_{A} .
$$


The Hermitian conjugation can be extended to higher valence space spinors by requiring that the conjugate of a product equals the product of conjugates. We also get

$$
\hat{\hat{\mu}}_{A_{1} \cdots A_{k}}=(-1)^{k} \hat{\mu}_{A_{1} \cdots A_{k}} .
$$

Furthermore, it is important to note

$$
\hat{\epsilon}_{A B}=\epsilon_{A B}, \quad \hat{\sigma}^{a}{ }_{A B}=-\sigma^{a}{ }_{A B} .
$$

\section{B. Basic variational formulae}

As in the case of standard spacetime spinors, we can compute the variations of the frames and the inverse metrics from the relations

$$
\begin{gathered}
\delta\left(e_{\mathbf{i}}{ }^{l}\right)=-e_{\mathbf{i}}^{j} e_{\mathbf{k}}{ }^{l} \delta \omega^{\mathbf{k}}{ }_{j}, \\
\delta\left(\epsilon_{\mathbf{A}}{ }^{D}\right)=-\epsilon_{\mathbf{A}}{ }^{B} \epsilon_{\mathbf{C}}{ }^{D} \delta \epsilon^{\mathbf{C}}{ }_{B}, \\
\delta\left(h^{k l}\right)=-\left(\delta h_{i j}\right) h^{i k} h^{j l}, \\
\delta\left(\epsilon^{C D}\right)=-\delta \epsilon_{A B} \epsilon^{A C} \epsilon^{B D} .
\end{gathered}
$$

Likewise, from relation (30a), we get

$$
\delta\left(\sigma_{A B}^{l}\right)=-\sigma^{k}{ }_{A B} \sigma_{C D}^{l} \delta \sigma_{k}{ }^{C D} .
$$

We can also split the variation of the coframes in terms of the variation of the metric and spin metric and gauge pieces,

$$
\begin{aligned}
\delta \omega^{\mathbf{m}}{ }_{a} & =-e_{\mathbf{h}}{ }^{b} h^{\mathbf{h m}} T_{a b}+\frac{1}{2} e_{\mathbf{h}}{ }^{b} h^{\mathbf{h m}} \delta h_{a b}, \\
\delta \epsilon^{\mathbf{P}}{ }_{A} & =-\epsilon_{\mathbf{H}}{ }^{B} \epsilon^{\mathbf{H P}} S_{A B}-\frac{1}{2} \epsilon_{\mathbf{H}}{ }^{B} \epsilon^{\mathbf{H P}} \delta \epsilon_{A B},
\end{aligned}
$$

where the tensor and spinor frame gauge fields are

$$
T_{a b} \equiv h_{\mathbf{c d}} \omega_{[a}^{\mathbf{d}} \delta \omega_{b]}^{\mathbf{c}}, \quad S_{A B} \equiv \omega_{(A}^{\mathbf{D}} \delta \omega_{B)}^{\mathbf{C}} \epsilon_{\mathbf{C D}} .
$$

A calculation following the same principles as for the spacetime version starting from relation (29a) gives the variation of the spatial soldering form,

$$
\delta \sigma_{k}^{A B}=-T_{k}{ }^{l}{\sigma_{l}}^{A B}+\frac{1}{2} \sigma^{l A B} \delta h_{k l}-2 \sigma_{k}^{(A|C|} S^{B)}{ }_{C}+\sigma_{k}^{(A|C|} \delta \epsilon^{B)}{ }_{C} .
$$

The irreducible parts are given by

$$
\begin{aligned}
\sigma^{k(C D} \delta \sigma_{k}{ }^{A B)} & =\frac{1}{2} \delta h^{(A B C D)}, \\
\sigma^{k(C}{ }_{B} \delta \sigma_{k}{ }^{A) B} & =T^{A C}-2 S^{A C}, \\
\sigma^{k}{ }_{C D} \delta \sigma_{k}{ }^{C D} & =\frac{1}{2} \delta h^{C D}{ }_{C D}+\frac{3}{2} \delta \epsilon_{C}^{C},
\end{aligned}
$$

where

$$
\begin{aligned}
T_{A B} & \equiv T_{k l} \sigma^{k}{ }_{A}^{C} \sigma_{B C}^{l}, \\
\delta h_{A B C D} & \equiv \sigma^{k}{ }_{A B} \sigma_{C D}^{l} \delta h_{k l} .
\end{aligned}
$$

We can now use this to see how the variation of vectors and covectors in space-spinor and tensor form differ,

$$
\begin{aligned}
& \sigma_{k}{ }^{A B} \delta \zeta^{k}=\delta\left(\zeta^{A B}\right)-\frac{1}{2} \delta \epsilon_{C}^{C} \zeta^{A B}-\frac{1}{2} \delta h^{A B}{ }_{C D} \zeta^{C D}+T^{(A|C|} \zeta^{B)}{ }_{C}-2 S^{(A|C|} \zeta^{B)}{ }_{C}, \\
& \sigma^{k}{ }_{A B} \delta \xi_{k}=\delta\left(\xi_{A B}\right)+\frac{1}{2} \delta \epsilon^{C}{ }_{C} \xi_{A B}+\frac{1}{2} \delta h_{A B C D} \xi^{C D}+T_{(A}{ }^{C} \xi_{B) C}-2 S_{(A}{ }^{C} \xi_{B) C},
\end{aligned}
$$

where $\zeta^{k}=\sigma^{k}{ }_{C D} \zeta^{C D}$ and $\xi_{k}=\sigma_{k}{ }^{C D} \zeta_{C D}$. This leads us to define a modified variation that cancels the gauge terms and the variation of the spin metric. 
Definition 2. For valence 1 space spinors, we define the modified variation operator $\vartheta$ via

$$
\begin{aligned}
& \vartheta\left(\phi_{A}\right) \equiv \delta\left(\phi_{A}\right)+\frac{1}{4} \delta \epsilon_{B}^{B} \phi_{A}+\frac{1}{2} T_{A}{ }^{B} \phi_{B}-S_{A}{ }^{B} \phi_{B}, \\
& \vartheta\left(\phi^{A}\right) \equiv \delta\left(\phi^{A}\right)-\frac{1}{4} \delta \epsilon^{B}{ }_{B} \phi^{A}-\frac{1}{2} T^{A}{ }_{B} \phi^{B}+S^{A}{ }_{B} \phi^{B} .
\end{aligned}
$$

These relations extend to higher valence spinors via the Leibnitz rule.

In the same way as for the spacetime variations, we get a relation between $\vartheta$ and spin frame component variation,

$$
\vartheta \phi_{A}=\epsilon_{A}^{\mathbf{B}} \delta\left(\phi_{\mathbf{B}}\right)+\frac{1}{2} T_{A}^{B} \phi_{B}
$$

The reality of $T_{a b}$ and (31) gives

$$
\widehat{T}_{A B}=T_{A B} .
$$

Expanding the frame index in Equation (32) and taking Hermitian conjugate yields

$$
\begin{aligned}
\widehat{\vartheta \phi}_{A} & =\epsilon^{\mathbf{1}}{ }_{A} \delta\left(\bar{\phi}_{\mathbf{0}^{\prime}}\right)-\epsilon^{\mathbf{0}}{ }_{A} \delta\left(\bar{\phi}_{\mathbf{1}^{\prime}}\right)+\frac{1}{2} T_{A}{ }^{B} \hat{\phi}_{B} \\
& =\epsilon^{\mathbf{0}}{ }_{A} \delta\left(\hat{\phi}_{\mathbf{0}}\right)+\epsilon^{\mathbf{1}}{ }_{A} \delta\left(\hat{\phi}_{\mathbf{1}}\right)+\frac{1}{2} T_{A}{ }^{B} \hat{\phi}_{B} \\
& =\vartheta(\hat{\phi})_{A} .
\end{aligned}
$$

Hence, the operation of Hermitian conjugation and the modified variation $\vartheta$ commute.

\section{Variations of the spatial connection}

Let $\mathcal{R}_{A B C D}$ denote the space spinor version of the trace free Ricci tensor, and let $\mathcal{R}$ be the Ricci scalar. Define

$$
\begin{aligned}
H^{A B C D} & \equiv \delta h^{(A B C D)}, \\
H & \equiv \delta h_{A B}^{A B}, \\
9_{A B C D} & \equiv-\frac{1}{2} D_{(C}^{F} \delta h_{D) F A B}, \\
F^{A B} & \equiv-\frac{1}{2} D^{A B} \delta h_{C D}^{C D}+D_{C D} \delta h^{A B C D} .
\end{aligned}
$$

Similarly to the case of spacetime spinors, we can compute the variation of a covariant derivative.

Theorem 3. The variation of a covariant space-spinor derivative is given by

$$
\vartheta\left(D_{A B} \kappa_{C}\right)=D_{A B} \vartheta \kappa_{C}+9_{A B C D} \kappa^{D}-\frac{1}{2} \delta h_{A B D F} D^{D F} \kappa_{C} .
$$

We also get

$$
\begin{aligned}
Q_{A}{ }^{C} B C & =-\frac{1}{6} D_{A B} H+\frac{1}{4} D_{C D} H_{A B}{ }^{C D}, \\
F_{A B} & =-\frac{1}{6} D_{A B} H+D_{C D} H_{A B}{ }^{C D}, \\
D_{D F} H_{A B C}{ }^{F} & =2 Q_{(A B C) D}+2 \epsilon_{D\left(A^{9}{ }_{B}{ }^{F} C\right) F}+\frac{1}{2} \epsilon_{D(A} D_{B C)} H .
\end{aligned}
$$

\section{Diffeomorphism dependence}

To analyse the dependence of the formalism on diffeomorphisms, we proceed in the same way as in Section IV C. Accordingly, let $\phi_{\lambda}$ be a one parameter group of diffeomorphisms generated by a vector field $\xi^{a}$. Now, let $h_{a b}[\lambda]=\phi_{-\lambda}^{*} \stackrel{\circ}{h}_{a b}$. All members of the family $h_{a b}[\lambda]$ will have the same geometric content and we get

$$
\left.\delta h_{a b}=\mathcal{L}_{\xi} h_{a b}=2 D_{(a} \xi_{b}\right)
$$


Moreover, one has that

$$
\begin{aligned}
Q_{A B C D} & =-\frac{1}{2} D_{(C}{ }^{F} D_{D) F} \xi_{A B}-\frac{1}{2} D_{(C}{ }^{F} D_{|A B|} \xi_{D) F}, \\
F^{A B} & =D_{C D} D^{C D} \xi^{A B}-\frac{1}{3} \mathcal{R} \xi^{A B}-\mathcal{R}^{A B}{ }_{C D} \xi^{C D} .
\end{aligned}
$$

Again, we see that $F^{A B}$ can be interpreted as a gauge source function for the linearised diffeomorphisms, but this time one needs to solve an elliptic equation instead of a wave equation to obtain $\xi^{A B}$ from $F^{A B}$.

\section{E. Variations of the spatial curvature}

By computing the variation of the commutator relations

$$
\begin{aligned}
\mathcal{R} \kappa_{A} & =8 D_{(A}{ }^{C} D_{B) C} K^{B}, \\
\mathcal{R}_{A B C D} K^{D} & \left.=2 D_{(A}{ }^{D} D_{B|D|} K_{C}\right),
\end{aligned}
$$

we get, after calculations similar to those carried out in the spacetime case, the variation of the curvature.

Theorem 4. The variation of the spatial curvature spinors are given by

$$
\begin{aligned}
\vartheta(\mathcal{R}) & =-\frac{1}{3} H \mathcal{R}-H^{B C D F} \mathcal{R}_{B C D F}-4 D_{C D}{ }^{Q^{B C}{ }_{B} D}, \\
\vartheta\left(\mathcal{R}_{A B C D}\right) & =-\frac{1}{12} H_{A B C D} \mathcal{R}-\frac{1}{3} H \mathcal{R}_{A B C D}+2 D_{(A}{ }^{F}{ }_{B|F| C D)}+\frac{1}{2} H_{(A B}{ }^{F H} \mathcal{R}_{C D) F H} .
\end{aligned}
$$

Proof. Computing the variation of relation (34a) gives

$$
\begin{aligned}
& \mathcal{R} \vartheta \kappa_{A}+\vartheta(\mathcal{R}) \kappa_{A}=-4 D_{A C} D_{B}^{C} \vartheta \kappa^{B}+\frac{4}{3} H D_{A C} D_{B}{ }^{C} \kappa^{B}+49_{B}{ }^{D}{ }_{C D} D_{A}{ }^{C} \kappa^{B}+4 \kappa^{B} D_{A D^{Q}}{ }^{C D}{ }_{B C} \\
& -2 H_{B C D F} D_{A}{ }^{F} D^{C D} K^{B}-\frac{2}{3} D_{A}{ }^{B} H D_{B C} K^{C}-4 D_{B C} D_{A}{ }^{C} \vartheta \kappa^{B} \\
& +\frac{4}{3} H D_{B C} D_{A}^{C} \kappa^{B}+49_{A}{ }^{D}{ }_{C D} D_{B}{ }^{C} K^{B}-2 H_{A C D F} D_{B}{ }^{F} D^{C D} \kappa^{B}
\end{aligned}
$$

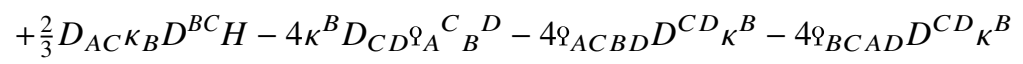

$$
\begin{aligned}
& +2 D_{A F} H_{B C D}{ }^{F} D^{C D} \kappa^{B}+2 D_{B F} H_{A C D}{ }^{F} D^{C D} K^{B}+2 H_{B C D F} D^{D F} D_{A}{ }^{C} K^{B} \\
& +2 H_{A C D F} D^{D F} D_{B}{ }^{C} K^{B} \\
& =\mathcal{R} \vartheta \kappa_{A}-\frac{1}{3} H \mathcal{R} \kappa_{A}-2 H_{A}{ }^{C D F} \mathcal{R}_{B C D F} \kappa^{B}+4 \kappa^{B} D_{A D^{Q}}{ }^{C D}{ }_{B C} \\
& -4 \kappa^{B} D_{C} D^{Q_{A}}{ }^{C}{ }_{B}{ }^{D} \\
& =\mathcal{R} \vartheta \kappa_{A}-\frac{1}{3} H \mathcal{R} \kappa_{A}-H^{B C D F} \mathcal{R}_{B C D F} \kappa_{A}-4 \kappa_{A} D_{C D} \mathrm{Q}^{B C}{ }_{B} D .
\end{aligned}
$$

Computing the variation of relation (34b) gives

$$
\begin{aligned}
& \left.\mathcal{R}_{A B C D} \vartheta K^{D}+\vartheta\left(\mathcal{R}_{A B C D}\right) K^{D}=2 D_{(A}{ }^{D} D_{B|D|} \vartheta \kappa_{C)}-\frac{2}{3} H D_{(A}{ }^{D} D_{B|D|} K_{C}\right) \\
& +H_{(A}{ }^{D F H} D_{B \mid D} D_{F H \mid} K_{C)}-H_{(A}{ }^{D F H} D_{|D F|} D_{B|H|} K_{C)} \\
& \left.-2 \mathrm{q}_{(A}{ }^{D}{ }_{B}{ }^{F} D_{|D F|} K_{C)}-2 \mathrm{Q}_{(A}{ }^{D}|D|^{F} D_{B|F|} K_{C}\right) \\
& \left.+2 \kappa^{D} D_{(A}{ }^{F} Q_{B|F| C) D}-\frac{1}{3} D_{(A}{ }^{D} H D_{B|D|} K_{C}\right) \\
& -D_{(A}{ }^{D} H_{B \mid D}{ }^{F H} D_{F H \mid} K_{C)} \\
& =\mathcal{R}_{A B C D} \vartheta \kappa^{D}-\frac{1}{12} H_{A B C D} \mathcal{R} \kappa^{D}-\frac{1}{3} H \mathcal{R}_{A B C D} K^{D} \\
& +2 \kappa^{D} D_{(A}{ }^{F} Q_{B|F| C) D}+\frac{1}{2} \kappa^{D} H_{(A B}{ }^{F H} \mathcal{R}_{C) D F H} \text {. }
\end{aligned}
$$


Remark 8. For pure gauge transformation (33), we get

$$
\begin{aligned}
\vartheta(\mathcal{R}) & =\mathcal{L}_{\xi} \mathcal{R}, \\
\vartheta\left(\mathcal{R}_{A B C D}\right) & =\mathcal{L}_{\xi} \mathcal{R}_{A B C D}-\mathcal{R}_{(A B}{ }^{F H} D_{C D)} \xi_{F H}-\mathcal{R}_{(A B}{ }^{F H} D_{|F H|} \xi_{C D)},
\end{aligned}
$$

where

$$
\mathcal{L}_{\xi} \mathcal{R}_{A B C D}=\xi^{F H} D_{F H} \mathcal{R}_{A B C D}+2 \mathcal{R}_{(A B}{ }^{F H} D_{C D)} \xi_{F H}
$$

\section{ACKNOWLEDGMENTS}

We thank L. B. Szabados for helpful conversations at the beginning of this project. We also thank L. Andersson and S. Aksteiner for discussions regarding gauge dependence. T.B. was supported by the Engineering and Physical Sciences Research Council [Grant No. EP/J011142/1].

\section{APPENDIX: ROTATIONS}

The purpose of this appendix is to discuss some issues related to the gauge in the frame and spin dyad formalisms.

\section{Lorentz transformations}

As it is well known, the metric $g_{a b}$ is not determined in a unique way by the orthonormal coframe $\omega_{a}^{\mathbf{a}}{ }_{a}$. Any other coframe related to $\omega^{\mathbf{a}}{ }_{a}$ by means of a Lorentz transformation, i.e., a matrix $\left(\Lambda_{\mathbf{b}}^{\mathbf{a}}\right)$ such that

$$
\eta_{\mathbf{a b}} \Lambda_{\mathbf{c}}^{\mathbf{a}} \Lambda_{\mathbf{d}}^{\mathbf{b}}=\eta_{\mathbf{c d}}
$$

It follows that $\grave{\omega}_{a}^{\mathbf{a}} \equiv \Lambda_{\mathbf{b}_{\mathbf{b}}} \omega^{\mathbf{b}}{ }_{a}$ is also orthonormal with respect to $g_{a b}$ and one can write $g_{a b}=$ $\eta_{\mathbf{a b}} \grave{\omega}^{\mathbf{a}}{ }_{a} \grave{\omega}_{b}^{\mathbf{b}}$. The associated orthonormal frame is $\grave{e}_{\mathbf{a}}{ }^{a}=\Lambda_{\mathbf{a}}{ }^{\mathbf{b}} e_{\mathbf{b}}{ }^{a}$ with $\left(\Lambda_{\mathbf{a}} \mathbf{b}\right) \equiv\left(\Lambda_{\mathbf{b}}^{\mathbf{a}}\right)^{-1}$ where the last expression is a relation between matrices.

The discussion in the previous paragraph can be extended to include spinors. Making use of the Infeld-van der Waerden symbols, Equation (A1) can be rewritten as

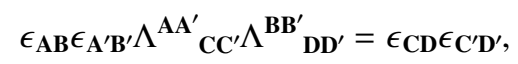

with $\Lambda^{\mathbf{A A}^{\prime}}{ }_{\mathbf{C C}^{\prime}} \equiv \sigma_{\mathbf{a}} \mathbf{A A}^{\prime} \sigma^{\mathbf{c}} \mathbf{C C}^{\prime} \Lambda^{\mathbf{a}}$. It can be shown that the spinorial components $\Lambda^{\mathbf{A A}^{\prime}}{ }_{\mathbf{C C}^{\prime}}$ can be decomposed as

$$
\Lambda_{\mathbf{C C}^{\prime}}^{\mathbf{A \mathbf { A } ^ { \prime }}}=\Lambda_{\mathbf{C}}^{\mathbf{A}} \bar{\Lambda}_{\mathbf{C}^{\prime}}^{\mathbf{A}^{\prime}}
$$

where $\left(\Lambda^{\mathbf{A}} \mathbf{C}\right)$ is a $S L(2, \mathbb{C})$ matrix. The latter naturally induces a change of spinorial basis via the relations

$$
\grave{\epsilon}_{\mathbf{A}}{ }^{A}=\Lambda_{\mathbf{A}}{ }^{\mathbf{B}} \epsilon_{\mathbf{B}}{ }^{A}, \quad \grave{\epsilon}_{A}^{\mathbf{A}}=\Lambda^{\mathbf{A}}{ }_{\mathbf{B}} \epsilon^{\mathbf{B}},
$$

with $\left(\Lambda_{\mathbf{A}}^{\mathbf{B}}\right) \equiv\left(\Lambda_{\mathbf{B}}^{\mathbf{A}}\right)^{-1}$. Crucially, one has that

$$
\epsilon_{\mathbf{A B}}=\Lambda_{\mathbf{A}}^{\mathbf{C}} \Lambda^{\mathbf{D}}{ }_{\mathbf{B}} \epsilon_{\mathbf{C D}}, \quad \epsilon^{\mathbf{A B}}=\Lambda_{\mathbf{C}}^{\mathbf{A}} \Lambda_{\mathbf{D}}^{\mathbf{B}} \epsilon^{\mathbf{C D}} .
$$

\section{2. $O(3)$-rotations}

Given a 3-dimensional negative-definite Riemannian metric $h_{i j}$ and an associated orthonormal coframe $\omega^{\mathbf{i}}{ }_{k}$, one has that

$$
h_{i j}=-\delta_{\mathbf{i j}} \omega_{i}^{\mathbf{i}} \omega_{j}^{\mathbf{j}} .
$$


Any other coframe $\grave{\omega}_{k}^{\mathbf{i}}$ related to the coframe $\omega^{\mathbf{i}}{ }_{k}$ through the relation $\grave{\omega}_{k}^{\mathbf{i}}=O_{\mathbf{j}} \mathbf{i} \omega^{\mathbf{j}}{ }_{k}$, where $\left(O_{\mathbf{j}}^{\mathbf{i}}\right)$ is a $O(3)$-matrix, gives rise to the same metric. The defining condition for $\left(O_{\mathbf{j}}^{\mathbf{i}}\right)$ can be expressed as

$$
\delta_{\mathbf{i j}}=\delta_{\mathbf{k l}} O_{\mathbf{i}}^{\mathbf{k}} O_{\mathbf{j}}{ }^{l}
$$

A direct calculation using the definition of the Hermitian product shows that the changes of spin dyad preserving the Hermitian structure induced by the Hermitian spinor $\varpi_{A A^{\prime}}$ are of the form $\grave{\epsilon}_{\mathbf{A}}{ }^{A}=O_{\mathbf{A}}{ }^{\mathbf{B}} \epsilon_{\mathbf{B}}{ }^{A}$ where $\left(O_{\mathbf{A}}{ }^{\mathbf{B}}\right)$ are $S U(2, \mathbb{C})$ matrices. As $S U(2, \mathbb{C})$ is a subgroup of $S L(2, \mathbb{C})$, one has that $\epsilon_{\mathbf{A B}}=O_{\mathbf{A}}{ }^{\mathbf{C}} O_{\mathbf{B}}{ }^{\mathbf{D}} \epsilon_{\mathbf{C D}}$. The matrices $\left(O_{\mathbf{j}}{ }^{\mathbf{i}}\right)$ and $\left(O_{\mathbf{A}}{ }^{\mathbf{B}}\right)$ are related to each other via the spatial Infeld-van der Waerden symbols,

$$
O_{\mathbf{i}}^{\mathbf{j}}=\sigma_{\mathbf{i}}^{\mathbf{A B}} \sigma^{\mathbf{j}} \mathbf{C D} O_{\mathbf{A}}{ }^{\mathbf{C}} O_{\mathbf{B}}{ }^{\mathbf{D}}
$$

${ }^{1}$ Arnowitt, R., Deser, S., and Misner, C. W., "The dynamics of general relativity," in Gravitation: An Introduction to Current Research, edited by Witten, L. (John Wiley \& Witten, 1962), p. 227.

${ }^{2}$ Bäckdahl, T., SymManipulator, 2011-2015, http://www.xact.es/SymManipulator.

${ }^{3}$ Bäckdahl, T. and Valiente Kroon, J. A., "Geometric invariant measuring the deviation from Kerr data," Phys. Rev. Lett. 104, 231102 (2010).

${ }^{4}$ Bäckdahl, T. and Valiente Kroon, J. A., "On the construction of a geometric invariant measuring the deviation from Kerr data," Ann. Henri Poincare 11, 1225 (2010).

${ }^{5}$ Bäckdahl, T. and Valiente Kroon, J. A., "Approximate twistors and positive mass," Classical Quantum Gravity 28, 075010 (2011).

${ }^{6}$ Dain, S., "Geometric inequalities for axially symmetric black holes," Classical Quantum Gravity 29, 73001 (2012).

${ }^{7}$ Geroch, R., Held, A., and Penrose, R., "A space-time calculus based on pairs of null directions," J. Math. Phys. 14, 874 (1973).

${ }^{8}$ Mars, M., "Present status of the Penrose inequality," Classical Quantum Gravity 26, 193001 (2009).

${ }^{9}$ Martín-García, J. M., xAct, 2002-2015, http://www.xact.es.

${ }^{10}$ Penrose, R. and Rindler, W., Spinors and Space-Time: Volume 1. Two-Spinor Calculus and Relativistic Fields (Cambridge University Press, 1984).

${ }^{11}$ The primed indices are moved up after the Lie derivative is taken to allow the symmetrizations to be written nicely.

${ }^{12}$ Troutman, J. L., Variational Calculus with Elementary Convexity (Springer Verlag, 1983).

${ }^{13}$ Witten, E., "A new proof of the positive energy theorem," Commun. Math. Phys. 80, 381 (1981). 